\title{
$G$-gerbes, principal 2-group bundles and characteristic classes
}

\author{
Grégory Ginot and Mathieu Stiénon
}

\begin{abstract}
Let $G$ be a Lie group and $G \rightarrow \operatorname{Aut}(G)$ be the canonical group homomorphism induced by the adjoint action of a group on itself. We give an explicit description of a 1-1 correspondence between Morita equivalence classes of, on the one hand, principal 2-group $[G \rightarrow \operatorname{Aut}(G)]$-bundles over Lie groupoids and, on the other hand, $G$-extensions of Lie groupoids (i.e. between principal $[G \rightarrow \operatorname{Aut}(G)]$ bundles over differentiable stacks and $G$-gerbes over differentiable stacks). This approach also allows us to identify $G$-bound gerbes and $[Z(G) \rightarrow 1]$-group bundles over differentiable stacks, where $Z(G)$ is the center of $G$. We also introduce universal characteristic classes for 2-group bundles. For groupoid central $G$-extensions, we introduce Dixmier-Douady classes that can be computed from connection-type data generalizing the ones for bundle gerbes. We prove that these classes coincide with universal characteristic classes. As a corollary, we obtain further that Dixmier-Douady classes are integral.
\end{abstract}

\section{Introduction}

This paper is devoted to the relation between groupoid $G$-extensions and principal Lie 2-group bundles and to their characteristic classes.

A Lie 2-group is a Lie groupoid $\Gamma_{2} \rightrightarrows \Gamma_{1}$, whose spaces of objects $\Gamma_{1}$ and of morphisms $\Gamma_{2}$ are Lie groups and all of whose structure maps are group morphisms. One shall note that in this paper we are interested in strict Lie 2 -groups only, though we believe all our results can be extended to weak ones as well. A crossed module $(G \stackrel{\rho}{\rightarrow} H)$ is a Lie group morphism $G \stackrel{\rho}{\rightarrow} H$ together with an action of $H$ on $G$ satisfying suitable compatibility conditions. It is standard that Lie 2-groups are in bijection with crossed modules [2, 15, 46]. In this paper, $[G \stackrel{\rho}{\rightarrow} H]$ denotes the 2-group corresponding to the crossed module $(G \stackrel{\rho}{\rightarrow} H)$. 
Lie 2-groups arise naturally in mathematical physics. For instance, in higher gauge theory [3, 5], Lie 2-group bundles provide a well suited framework for describing the parallel transport of strings [1, 3, 38]. Several recent works have approached the concept of bundles with a "structure Lie 2-group" over a manifold from various perspectives [1, 3, 6, 50, 58. Here we take an alternative point of view and give a definition of principal Lie 2-group bundles of a global nature (i.e. not resorting to a description explicitly involving local charts and cocycles) and which allows for the base space to be a Lie groupoid. In other words, we consider 2-group principal bundles over differentiable stacks [8]. Our approach immediately leads to a natural construction of "universal characteristic classes" for principal 2-group bundles.

Let us start with Lie (1-)groups. A principal $G$-bundle $P$ over a manifold $M$ canonically determines a homotopy class of maps from $M$ to the classifying space $B G$ of the group $G$. In fact, the set of isomorphism classes of $G$-principal bundles over $M$ is in bijection with the set of homotopy classes of maps $M \stackrel{f}{\rightarrow} B G\left[17\right.$, 53, 54]. Pulling back the generators of $H^{*}(B G)$ (the universal classes) through $f$, one obtains characteristic classes of the principal bundle $P$ over $M$. These characteristic classes coincide with those obtained from a connection by applying the Chern-Weil construction [21, 42,

There is an analogue but much less known, differential geometric rather than purely topological, point of view: a principal $G$-bundle over a manifold $M$ can be thought of as a "generalized morphism" (in the sense of Hilsum \& Skandalis [30, 47]) from the manifold $M$ to the Lie group $G$ both considered as 1-groupoids. To see this, recall that a principal $G$-bundle can be defined as a collection of transition functions $g_{i j}: U_{i j} \rightarrow G$ on the double intersections $U_{i j}$ of some open covering $\left\{U_{i}\right\}_{i \in I}$ of $M$, satisfying the cocycle condition $g_{i j} g_{j k}=g_{i k}$. These transition functions constitute a morphism of groupoids from the Čech groupoid $\coprod U_{i j} \rightrightarrows \coprod U_{i}$ associated to the open covering $\left\{U_{i}\right\}_{i \in I}$ to the Lie group $G \rightrightarrows *$. Hence we have a diagram

$$
(M \rightrightarrows M) \stackrel{\sim}{\longleftarrow}\left(\coprod U_{i j} \rightrightarrows \coprod U_{i}\right) \rightarrow(G \rightrightarrows *)
$$

in the category of Lie groupoids and their morphisms whose leftward arrow is a Morita equivalence, in other words a generalized morphism from the manifold $M$ to the Lie group $G$.

This second point of view, or more precisely its generalization to the 2groupoid context, constitutes the foundation on which our approach is built. The generalization of the concept of "generalized morphism" to 2-groupoids is straightforward: a generalized morphism of Lie 2-groupoids $\boldsymbol{\Gamma} \rightsquigarrow \boldsymbol{\Delta}$ is a 
diagram $\boldsymbol{\Gamma} \underset{\sim}{\stackrel{\phi}{\sim}} \boldsymbol{E} \stackrel{f}{\rightarrow} \boldsymbol{\Delta}$ in the category $\mathbf{2 G p d}$ of Lie 2-groupoids and their morphisms, where $\phi$ is a Morita equivalence (a "smooth" equivalence of 2-groupoids). It is sometimes useful to think of two Morita equivalent Lie 2 -groupoids as two different choices of an atlas (or open cover) on the same geometric object (which is a differentiable 2-stack [8, 13]). We define a principal $[G \stackrel{\rho}{\rightarrow} H]$-bundle over a Lie groupoid $\Gamma$ to be a generalized morphism from $\Gamma$ to $[G \stackrel{\rho}{\rightarrow} H]$ (up to equivalence). See Section 2.3 .

The concept of (geometric) nerve of Lie groupoids extends to the 2categorical context as a functor from the category of Lie 2-groupoids to the category of simplicial manifolds [55]. By convention, the cohomology of a 2-groupoid is the cohomology of its nerve, which can be computed via a double complex (for instance, see [25]). Crucially, Morita equivalences induce isomorphisms in cohomology. Therefore, any generalized morphism of 2-groupoids $\Gamma \stackrel{F}{\rightsquigarrow}[G \rightarrow H]$ defining a principal $[G \rightarrow H]$-bundle $\mathfrak{B}$ over the groupoid $\Gamma$ yields a pullback homorphism $F^{*}: H^{\bullet}([G \rightarrow H]) \rightarrow H^{\bullet}(\Gamma)$ in cohomology, which is called the cohomology characteristic map (characteristic map for short). The cohomology classes in $H^{\bullet}([G \rightarrow H])$ [25] should be viewed as universal characteristic classes and their images by $F^{*}$ as the characteristic classes of $\mathfrak{B}$.

Lie 2-group principal bundles are closely related to non-abelian gerbes. Geometrically, non-abelian $G$-gerbes over differentiable stacks can be considered as groupoid $G$-extensions modulo Morita equivalence [33]. By a groupoid $G$-extension, we mean a short exact sequence of groupoids $1 \rightarrow$ $M \times G \stackrel{i}{\rightarrow} \tilde{\Gamma} \stackrel{\phi}{\rightarrow} \Gamma \rightarrow 1$, where $M \times G$ is a bundle of groups.

One of our main results is an equivalence between (strict) $G$-gerbes over a differentiable stack and principal bundles over the 2-group $[G \rightarrow \operatorname{Aut}(G)]$. More precisely, we establish an explicit 1-1 correspondence between groupoid $G$-extensions up to Morita equivalence (i.e. strict $G$-gerbes over differentiable stacks) and principal $[G \rightarrow \operatorname{Aut}(G)]$-bundles over Lie groupoids modulo Morita equivalence (i.e. $[G \rightarrow \operatorname{Aut}(G)]$-principal bundles over differentiable stacks). This is Theorem 3.4. Note that a restricted version of this correspondence is highlighted in [34, Theorem 4].

It is known that Giraud's second non abelian cohomology group $H^{2}(\mathfrak{X}, G)$ classifies the $G$-gerbes over a differentiable stack $\mathfrak{X}$ [26] while Dedecker's $H^{1}(\mathfrak{X},[G \rightarrow \operatorname{Aut}(G)])$ classifies the principal $[G \rightarrow \operatorname{Aut}(G)]$-bundles [18, 19]. In [12, 14], Breen showed that these two cohomology groups are isomorphic. In some sense, our theorem above can be considered as an explicit geometric proof of Breen's theorem in the smooth context. Indeed, one of the main motivations behind the present paper is the relation between $G$-extensions 
and 2-group principal bundles. We believe our result throws a bridge between the groupoid extension approach to the differential geometry of $G$-gerbes developed in [33] and the one based on higher gauge theory due to Baez \& Schreiber [3]. This will be investigated somewhere else.

An important class of $G$-extensions is formed by the so called central $G$ extensions [33, those for which the structure 2-group $[G \rightarrow \operatorname{Aut}(G)]$ reduces to the 2-group $[Z(G) \rightarrow 1]$ (where $Z(G)$ stands for the center of $G$ ). They correspond to $G$-gerbes with trivial band or $G$-bound gerbes [33]. Each such extension determines a principal $[Z(G) \rightarrow 1]$-bundle over the base groupoid $\Gamma$. In [8], Behrend \& Xu gave a natural construction associating a class in $H^{3}(\Gamma)$ to a central $S^{1}$-extension of a Lie groupoid $\Gamma$. When the base Lie groupoid is Morita equivalent to a smooth manifold (viewed as a trivial 2-groupoid), a central $S^{1}$-extension is what has been studied by Murray and Hitchin under the name bundle gerbe [31, 45]. The Behrend-Xu class of a bundle gerbe coincides with its Dixmier-Douady class, which can be described by the 3 -curvature. In the present paper, we extend the construction of Behrend \& Xu and define a Dixmier-Douady class $\boldsymbol{D} \boldsymbol{D}_{(\alpha)} \in H^{3}(\Gamma) \otimes$ $Z(\mathfrak{g})$ for any central $G$-extension, where $G$ is connected with a reductive Lie algebra. Since a central $G$-extension induces a $[Z(G) \rightarrow 1]$-principal bundle over $\Gamma$, there is also a charateristic map $H^{3}([Z(G) \rightarrow 1]) \rightarrow H^{3}(\Gamma)$. Dualizing, one obtains a class $\boldsymbol{C C}_{\phi} \in H^{3}(\Gamma) \otimes Z(\mathfrak{g})$. We prove that the DixmierDouady class $\boldsymbol{D} \boldsymbol{D}_{(\alpha)}$ coincides with the characteristic class $\boldsymbol{C} \boldsymbol{C}_{\phi}$. In a certain sense, this is the gerbe analogue of the Chern-Weil isomorphism for principal bundles [21, 42].

The paper is organized as follows. Section 2 is devoted to generalized morphisms of Lie 2-groupoids and to 2-group bundles and recalls some standard material on Lie 2-groupoids. The main feature of Section 3 is Theorem 3.4 on the equivalence of groupoids $G$-extensions and principal $[G \stackrel{\mathrm{Ad}}{\longrightarrow}$ Aut $(G)$ ]-bundles. In Section 4 we define the characteristic map/classes of principal Lie 2-group bundles, we present the construction of the DixmierDouady classes of groupoid central $G$-extensions and we prove that the Dixmier-Douady class of a central $G$-extension coincides with the universal characteristic class of the induced $[Z(G) \rightarrow 1$ ]-bundle - see Theorem 4.18. Since the universal characteristic map can be defined in cohomology with integer coefficients, we obtain that the Dixmier-Douady class of a central $G$-extension is integral as a corollary of our study. This applies, in particular, in the classical case of a bundle gerbe.

Note that, when $G$ is discrete, the relation between groupoid $G$-extensions and 2-group principal bundles was also independently studied by Haefliger [27, 28. 
Some of the results of the present paper are related to results announced by Baez \& Stevenson [4]. Recently, Sati, Stasheff \& Schreiber have studied characteristic classes for 2-group bundles by the mean of $L_{\infty}$-algebras [50]. It would be very interesting to relate their construction to ours using integration of $L_{\infty}$-algebras as in [23, 29].

\section{Generalized morphisms and principal Lie 2-group bundles}

\subsection{Lie 2-groupoids, Crossed modules and Morita morphisms}

This section is concerned with Lie 2-groupoids and Morita equivalences. The material is rather standard. For instance, see [39, 44] for the general theory of Lie groupoids and [2, 46, 60] for Lie 2-groupoids. We only deal with the case of strict 2 -groupoids and strict 2 -groups.

Definition 2.1. A Lie 2-groupoid is a double Lie groupoid

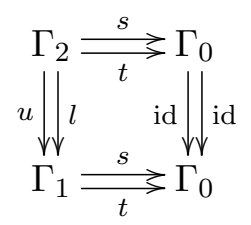

in the sense of [15], where the right column $\Gamma_{0} \underset{\mathrm{id}}{\stackrel{\mathrm{id}}{\rightleftarrows}} \Gamma_{0}$ denotes the trivial groupoid associated to the smooth manifold $\Gamma_{0}$. It makes sense to use the symbols $s$ and $t$ to denote the source and target maps of the groupoid $\Gamma_{2} \rightrightarrows \Gamma_{0}$ since $s \circ l=s \circ u$ and $t \circ l=t \circ u$.

Remark 2.2. A Lie 2-groupoid is thus a small 2-category in which all arrows are invertible, the sets of objects, 1-arrows and 2-arrows are smooth manifolds, all structure maps are smooth and the sources and targets are surjective submersions.

In the sequel, the 2-groupoid (1) will be denoted $\Gamma_{2} \frac{l}{u} \geq \Gamma_{1} \frac{s}{t} \geq \Gamma_{0}$ or just $\boldsymbol{\Gamma}$. The so called vertical (resp. horizontal) multiplication in the groupoid $\Gamma_{2} \underset{u}{\stackrel{l}{\rightleftarrows}} \Gamma_{1} \quad\left(\right.$ resp. $\left.\Gamma_{2} \underset{t}{\stackrel{s}{\rightleftarrows}} \Gamma_{0}\right)$ will be denoted by $\star($ resp. *)

Clearly, a Lie groupoid can be seen as a Lie 2-groupoid $\Gamma_{1} \underset{\text { id }}{\stackrel{\text { id }}{\longrightarrow}} \ \Gamma_{1} \underset{t}{\stackrel{s}{\gtrless}} \Gamma_{0}$. 
A Lie 2-groupoid where $\Gamma_{0}$ is the one-point space $*$ is known as a Lie 2-group.

There is a well-known equivalence between Lie 2-groupoids and crossed modules of groupoids [15].

Definition 2.3. A crossed module of groupoids $(X \stackrel{\rho}{\rightarrow} \Gamma)$ is a morphism of groupoids

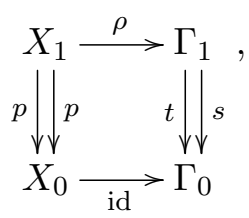

from a family of groups $X_{1} \stackrel{p}{\rightarrow} X_{0}$ to a groupoid $\Gamma_{1} \rightrightarrows \Gamma_{0}$ sharing the same unit space $X_{0}=\Gamma_{0}$, together with a right action by automorphisms $(\gamma, x) \mapsto$ $x^{\gamma}$ of $\Gamma_{1} \rightrightarrows \Gamma_{0}$ on $X_{1} \rightarrow X_{0}$ satisfying:

$$
\begin{array}{rlrl}
\rho\left(x^{\gamma}\right) & =\gamma^{-1} \rho(x) \gamma & & \forall(x, \gamma) \in X_{1} \times_{\Gamma_{0}} \Gamma_{1}, \\
x^{\rho(y)} & =y^{-1} x y & \forall(x, y) \in X_{1} \times_{\Gamma_{0}} X_{1} .
\end{array}
$$

Note that the equalities (2) and (3) make sense because $X_{1}$ is a family of groups.

Example 2.4. Given any Lie group $G$, we obtain a crossed module by setting $X_{1}=G, \Gamma_{1}=\operatorname{Aut}(G), \Gamma_{0}=*$ and $\rho(g)=\operatorname{Ad}_{g}$ (the conjugation by $g$ ).

Example 2.5. A Lie groupoid $\Gamma_{1} \rightrightarrows \Gamma_{0}$ induces a crossed module in the following way. Let $S_{\Gamma}=\left\{x \in \Gamma_{1} \mid s(x)=t(x)\right\}$ be the set of closed loops in $\Gamma_{1}$. Then $S_{\Gamma}$ is a family of groups over $\Gamma_{0}$ and $\Gamma_{1}$ acts by conjugation on $S_{\Gamma}$. Therefore, we obtain a crossed module

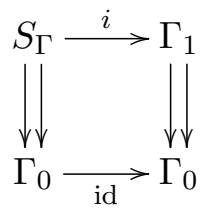

where $i$ is the inclusion map.

A 2-groupoid $\Gamma_{2} \underset{u}{\stackrel{l}{\rightleftarrows}} \Gamma_{1} \underset{t}{\stackrel{s}{\rightrightarrows}} \Gamma_{0}$ determines a crossed module of groupoids $(G \stackrel{\rho}{\rightarrow} H)$ as follows. Here the groupoid $H$ is $\Gamma_{1} \rightrightarrows \Gamma_{0}, G_{1}=$ 
$\left\{g \in \Gamma_{2} \mid l(g) \in \Gamma_{0} \subset \Gamma_{1}\right\}, \rho$ is the restriction of $u$ to $G_{1}$ and the action of $H_{1}=\Gamma_{1}$ on $G_{1} \subset \Gamma_{2}$ is by conjugation. More precisely, if $1_{h}$ is the unit over an object $h$ in the groupoid $\Gamma_{2} \stackrel{l}{\stackrel{l}{u}} \geqslant \Gamma_{1}$, then $g^{h}=1_{h^{-1}} * g * 1_{h}$. Conversely, given a crossed module of groupoids $(X \stackrel{\rho}{\rightarrow} \Gamma)$, one gets a Lie 2groupoid $X_{1} \ltimes \Gamma_{1} \stackrel{l}{\vec{u}} \underset{\Gamma_{1}}{\stackrel{s}{\rightleftarrows}} \Gamma_{0}$, where $X_{1} \ltimes \Gamma_{1} \rightrightarrows \Gamma_{1}$ is the transformation groupoid and $X_{1} \ltimes \Gamma_{1} \rightrightarrows \Gamma_{0}$ is the semi-direct product of groupoids. More precisely, for all $x, x^{\prime} \in X_{1}$ and $\gamma, \gamma^{\prime} \in \Gamma_{1}$, the structures maps are defined by

$$
\begin{array}{ll}
l(x, \gamma)=\gamma, & \left(x^{\prime}, \gamma^{\prime}\right) *(x, \gamma)=\left(x^{\prime} x^{\gamma^{\prime-1}}, \gamma^{\prime} \gamma\right) \\
u(x, \gamma)=\rho(x) \gamma, & \left(x^{\prime}, \rho(x) \gamma\right) \star(x, \gamma)=\left(x^{\prime} x, \gamma\right) .
\end{array}
$$

In the sequel, we will denote the Lie 2-groupoid associated to the crossed module $(G \stackrel{\rho}{\rightarrow} H)$ by $[G \stackrel{\rho}{\rightarrow} H]$.

Example 2.6. The crossed module of groups $(G \stackrel{\text { Ad }}{\longrightarrow} \operatorname{Aut}(G))$ yields the 2-group $G \ltimes \operatorname{Aut}(G) \stackrel{l}{u} \underset{u u t}{\longrightarrow}(G) \Longrightarrow *$ with structure maps

$$
\begin{gathered}
l(g, \varphi)=\varphi \quad u(g, \phi)=\mathbf{A d}_{g} \circ \varphi \\
\left(g_{1}, \mathbf{A d}_{g_{2}} \circ \varphi_{2}\right) \star\left(g_{2}, \varphi_{2}\right)=\left(g_{1} g_{2}, \varphi_{2}\right) \\
\left(g_{1}, \varphi_{1}\right) *\left(g_{2}, \varphi_{2}\right)=\left(g_{1} \varphi_{1}\left(g_{2}\right), \varphi_{1} \circ \varphi_{2}\right)
\end{gathered}
$$

A (strict) morphism $\boldsymbol{\Gamma} \stackrel{\phi}{\rightarrow} \boldsymbol{\Delta}$ of Lie 2-groupoids is a triple $\left(\phi_{0}, \phi_{1}, \phi_{2}\right)$ of smooth maps $\phi_{i}: \Gamma_{i} \rightarrow \Delta_{i}(i=0,1,2)$ commuting with all structure maps. Morphisms of crossed modules are defined similarly.

Let $\Delta$ be a Lie 2 -groupoid. Given a surjective submersion $f: M \rightarrow \Delta_{0}$, we can form the pullback Lie 2-groupoid

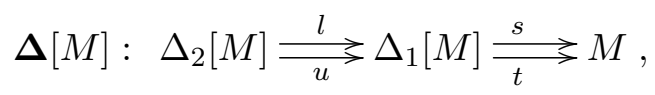

where

$$
\Delta_{i}[M]=\left\{(m, \gamma, n) \in M \times \Delta_{i} \times M \text { s.t. } s(\gamma)=f(m), t(\gamma)=f(n)\right\},
$$

for $i \in\{1,2\}$. The maps $s, t$ are the projections on the first and last factor respectively. The maps $u, l$, the horizontal and vertical multiplications are 
induced by the ones on $\boldsymbol{\Delta}$ as follows:

$$
\begin{array}{ll}
u(m, \gamma, n)=(m, u(\gamma), n), & (m, \gamma, n) *\left(n, \gamma^{\prime}, p\right)=\left(m, \gamma * \gamma^{\prime}, p\right), \\
l(m, \gamma, n)=(m, l(\gamma), n), & (m, \gamma, n) \star\left(m, \gamma^{\prime}, n\right)=\left(m, \gamma \star \gamma^{\prime}, n\right) .
\end{array}
$$

There is a natural map of groupoids $\boldsymbol{\Delta}[M] \rightarrow \boldsymbol{\Delta}$ defined by $m \mapsto f(m)$ and $(m, \gamma, n) \mapsto \gamma$.

Pullback of 2-groupoids yield a convenient definition of a higher analogue for Lie 2-groupoids of the notion of Morita morphism or weak equivalence of Lie 1-groupoids. These maps are higher analogues of the notion of a cover and thus are called hypercovers to agree with the terminology of [59, 60].

Definition 2.7. A morphism of Lie 2-groupoids $\boldsymbol{\Gamma} \stackrel{\phi}{\rightarrow} \boldsymbol{\Delta}$ is a hypercover if $\phi$ is the composition of two morphisms

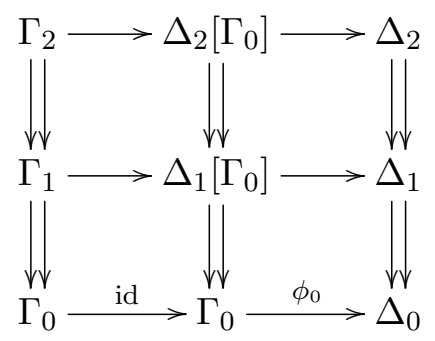

such that $\Gamma_{0} \rightarrow \Delta_{0}$ and $\Gamma_{1} \rightarrow \Delta_{1}\left[\Gamma_{0}\right]$ are surjective submersions and

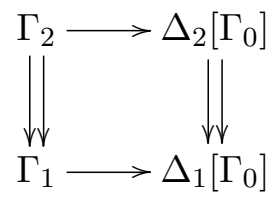

is a Morita morphism 1 bf 1 -groupoids.

Definition 2.8. The (weakest) equivalence relation generated by the hypercovers is called Morita equivalence $2^{2}$ More precisely, two Lie 2-groupoids $\boldsymbol{\Gamma}$ and $\boldsymbol{\Delta}$ are Morita equivalent if there exists a finite collection $\boldsymbol{E}_{0}, \boldsymbol{E}_{1}, \ldots, \boldsymbol{E}_{n}$ of Lie 2-groupoids with $\boldsymbol{E}_{0}=\boldsymbol{\Gamma}$ and $\boldsymbol{E}_{n}=\boldsymbol{\Delta}$, and, for each $i \in\{1, \ldots, n\}$, either a hypercover $\boldsymbol{E}_{i-1} \stackrel{\sim}{\rightarrow} \boldsymbol{E}_{i}$ or a hypercover $\boldsymbol{E}_{i} \stackrel{\sim}{\rightarrow} \boldsymbol{E}_{i-1}$.

\footnotetext{
${ }^{1}$ Here we follow the terminology of $[8,33$. Moerdijk calls such maps weak equivalences 44.

${ }^{2}$ By analogy with [8], we will sometimes refer to a hypercover as a Morita morphism since it induces a Morita equivalence.
} 
In fact, by Lemma 2.174, , one has the following well-known lemma.

Lemma 2.9. If $\boldsymbol{\Gamma}$ and $\boldsymbol{\Delta}$ are Morita equivalent, there exits a chain of hypercovers $\boldsymbol{\Gamma} \stackrel{\sim}{\leftarrow} \boldsymbol{E} \stackrel{\sim}{\rightarrow} \boldsymbol{\Delta}$ of length 2 in between $\boldsymbol{\Gamma}$ and $\boldsymbol{\Delta}$.

Remark 2.10. From the categorical point of view, a hypercover $\phi: \Gamma \stackrel{\sim}{\rightarrow}$ $\boldsymbol{\Delta}$ is in particular a 2-equivalence of 2-categories preserving the smooth structures.

We expect that the notion of Morita equivalence introduced here will help shed light on the integration problem for Courant algebroids [37] and more specifically on the relation between the different proposed approaches [36, 40, 52]. It is expected that the objects integrating Courant algebroids are symplectic 2-stacks [41.

Remark 2.11. Similar to [8], one can define differentiable 2-stacks. Two Lie 2-groupoids define the same differentiable 2-stack if, and only if, they are Morita equivalent. In fact a Lie 2-groupoid can be thought of as a choice of a differentiable atlas on a differentiable 2-stack.

\subsection{Generalized morphisms of Lie 2-groupoids}

Generalized morphisms of Lie 2-groupoids are a straightforward generalization of generalized morphisms of Lie (1-)groupoids [30, 44]. They also have been considered in [60]. Let $\mathbf{2 G p d}$ denote the category of Lie 2-groupoids and morphisms of Lie 2-groupoids.

Definition 2.12. A generalized morphism $F$ is a zigzag

$$
\boldsymbol{\Gamma} \leftarrow \boldsymbol{E}_{1} \rightarrow \cdots \stackrel{\sim}{\leftarrow} \boldsymbol{E}_{n} \rightarrow \boldsymbol{\Delta}
$$

where all leftward arrows are hypercovers. We use a squig arrow $F: \boldsymbol{\Gamma} \rightsquigarrow$ $\boldsymbol{\Delta}$ to denote a generalized morphism. The composition of two generalized morphisms is defined by the concatenation of two zigzags.

In fact we are interested in equivalence classes of generalized morphisms:

In the sequel, we will consider two morphisms of 2-groupoids $f: \boldsymbol{\Gamma} \rightarrow$ $\boldsymbol{\Delta}$ and $g: \boldsymbol{\Gamma} \rightarrow \boldsymbol{\Delta}$ to be equivalent if there exists two smooth applications $\varphi: \Gamma_{0} \rightarrow \Delta_{1}$ and $\psi: \Gamma_{1} \rightarrow \Delta_{2}$ such that, for any $x \in \Gamma_{2}$ and any pair of 
composable arrows $i, j \in \Gamma_{1}$, the following relations are satisfied:

$$
\begin{gathered}
\left(g_{2}(x) * 1_{\varphi(s(x))}\right) \star \psi(l(x))=\psi(u(x)) \star\left(1_{\varphi(t(x))} * f_{2}(x)\right), \\
\psi(j * i)=\left(1_{g_{1}(j)} * \psi(i)\right) \star\left(\psi(j) * 1_{f_{1}(i)}\right) .
\end{gathered}
$$

In other words, $f$ and $g$ are "conjugate" by a (invertible) map $\psi$ compatible with the horizontal multiplication.

It is easy to check that the conditions (4) and (5) are equivalent to the data of a natural 2-transformation from $f$ to $g$ [11, 35. Recall that a natural 2-transformation is given by the following data: an arrow $\varphi(m) \in \Delta_{1}$ for each object $m \in \Gamma_{0}$, and a 2-arrow $\psi(\gamma) \in \Delta_{2}$ for each arrow $\gamma \in \Gamma_{1}$ as in the diagram

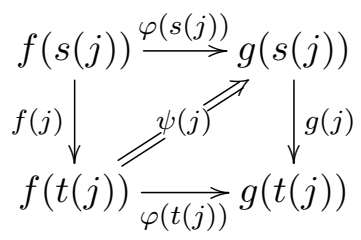

and satisfying obvious compatibility conditions with respect to the compositions of arrows and 2-arrows.

We now introduce the notion of equivalence of generalized morphisms; it is the natural equivalence relation on generalized morphisms extending the equivalence of groupoids morphisms.

Definition 2.13. Equivalence of generalized morphisms is the weakest equivalence relation satisfying the following three properties:

1) If there exists a natural transformation between a pair $f, g$ of homomorphisms of 2-groupoids, $f$ and $g$ are equivalent as generalized morphisms.

2) If $\boldsymbol{\Gamma} \stackrel{\phi}{\rightarrow} \boldsymbol{\Delta}$ is a hypercover of 2-groupoids, the generalized morphisms $\boldsymbol{\Delta} \stackrel{\phi}{\leftarrow} \boldsymbol{\Gamma} \stackrel{\phi}{\rightarrow} \boldsymbol{\Delta}$ and $\boldsymbol{\Gamma} \stackrel{\phi}{\rightarrow} \boldsymbol{\Delta} \stackrel{\phi}{\leftarrow} \boldsymbol{\Gamma}$ are equivalent to $\boldsymbol{\Delta} \stackrel{\text { id }}{\rightarrow} \boldsymbol{\Delta}$ and $\boldsymbol{\Gamma} \stackrel{\text { id }}{\longrightarrow} \boldsymbol{\Gamma}$, respectively.

3) Pre- and post-composition with a third generalized morphism preserves the equivalence.

Generalized morphisms can be seen as the 1-morphisms in a bicategory of fractions $\mathbf{2} \mathbf{G p d}\left[\mathcal{M}^{-1}\right]$, where we have "formally inverted" the collection $\mathcal{M}$ of hypercovers, and equivalence between generalized morphisms as being 
(invertible) 2-morphisms. We refer to [32, Chapter 7] for details on localization of categories with respect to a multiplicative system (see Lemma 2.17 below) and to [48, Chapter 3] and [49, Section 2] for details on the construction of the two cells of the associated bicategory of fractions. In particular, the 2-morphisms in the bicategory of fractions $\mathbf{2} \mathbf{G p d}\left[\mathcal{M}^{-1}\right]$ are represented by diagrams

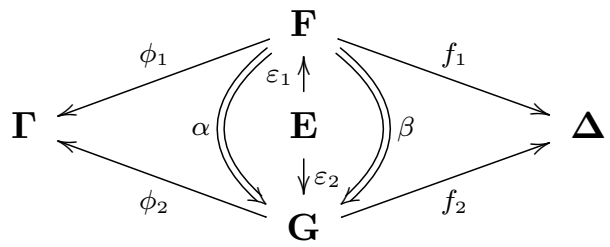

in which $\phi_{i} \circ \varepsilon_{i}$ are Morita morphisms (i.e. hypercovers) and $\alpha, \beta$ are 2transformations as above. See [48, Section 3.2.3] or [49, Section 2].

However, we will essentially only need the 1-category underlying $\mathbf{2} \operatorname{Gpd}\left[\mathcal{M}^{-1}\right]$.

Example 2.14. Let $F_{1}: \boldsymbol{\Gamma} \underset{\sim}{\stackrel{\phi_{1}}{\sim}} \boldsymbol{E}_{\mathbf{1}} \stackrel{f_{1}}{\longrightarrow} \boldsymbol{\Delta}$ and $F_{2}: \boldsymbol{\Gamma} \stackrel{\phi_{2}}{\sim} \boldsymbol{E}_{\mathbf{2}} \stackrel{f_{2}}{\longrightarrow} \boldsymbol{\Delta}$ be two generalized morphisms. Suppose that there exists a morphism $\mathbf{E}_{\mathbf{1}} \stackrel{\varepsilon}{\rightarrow} \mathbf{E}_{\mathbf{2}}$ such that the diagram

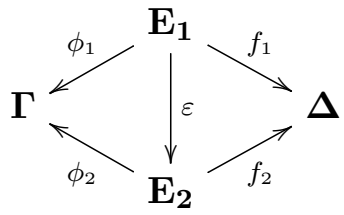

commutes up to 2-transformations (in particular, $\phi_{2} \circ \varepsilon$ is an hypercover). Then $F_{1}$ and $F_{2}$ are equivalent generalized morphisms.

Example 2.15. By its very definition, a Morita equivalence of groupoids $\boldsymbol{\Gamma} \stackrel{\sim}{\leftarrow} \mathbf{E}_{1} \stackrel{\sim}{\rightarrow} \cdots \stackrel{\sim}{\leftarrow} \mathbf{E}_{n} \stackrel{\sim}{\rightarrow} \boldsymbol{\Delta}$ defines two generalized morphisms $F: \boldsymbol{\Gamma} \rightsquigarrow \boldsymbol{\Delta}$ and $G: \boldsymbol{\Delta} \rightsquigarrow \boldsymbol{\Gamma}$. The compositions $F \circ G$ and $G \circ F$ are both equivalent to the identity.

Remark 2.16. As previously mentionned, generalized morphisms are obtained by formally inverting the Morita morphisms (i.e. hypercovers). In fact, the following Lemma can be checked.

Lemma 2.17. The collection $\mathcal{M}$ of all hypercovers of Lie 2-groupoids is a left multiplicative system [32, Definition 7.1.5] [56, Definition 10.3.4] in $\mathbf{2 G p d}$. Indeed, the following properties hold: 
1) $(\boldsymbol{\Gamma} \stackrel{\text { id }}{\rightarrow} \boldsymbol{\Gamma}) \in \mathcal{M}, \forall \boldsymbol{\Gamma} \in \mathbf{2} \mathbf{G p d}$;

2) $\mathcal{M}$ is closed under composition;

3) given $\boldsymbol{\Gamma} \stackrel{f}{\rightarrow} \boldsymbol{\Delta} \stackrel{\phi}{\stackrel{\sim}{\sim}} \boldsymbol{E}$ in $\mathbf{2 G p d}$ with $\phi \in \mathcal{M}$, there exists $\boldsymbol{\Gamma} \stackrel{\psi}{\sim} \boldsymbol{Z} \stackrel{g}{\rightarrow} \boldsymbol{E}$ in 2Gpd with $\psi \in \mathcal{M}$ such that

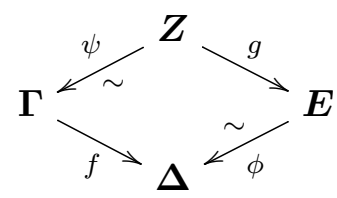

commutes;

4) given $\boldsymbol{\Gamma} \underset{\sim}{\stackrel{\phi}{\sim}} \boldsymbol{\Delta} \underset{g}{\stackrel{f}{\rightleftarrows}} \boldsymbol{E}$ in $\mathbf{2 G p d}$ with $\phi \in \mathcal{M}, f \circ \phi=g \circ \phi$ implies $f=g$.

Proof. Properties 20 and 3) follow from [59, Theorem 2.12] or [60, Section 2]. Property 4 follows from the fact that the map $\phi_{0}: \Gamma_{0} \rightarrow \Delta_{0}$ is a surjective submersion and that $\phi$ is an equivalence of 2-categories.

Since $\mathcal{M}$ is a left multiplicative system in the category $\mathbf{2} \mathbf{G p d}$, we can consider the localization $\mathbf{2} \mathbf{G p d}_{\mathcal{M}}$ of $\mathbf{2} \mathbf{G p d}$ with respect to $\mathcal{M}$ [32, Chapter 7] [56, Section 10.3]. This new category $\mathbf{2} \mathbf{G p d}_{\mathcal{M}}$ has the same objects as 2Gpd but its arrows are equivalence classes of generalized morphisms. An isomorphism in $\mathbf{2} \mathbf{G p d}_{\mathcal{M}}$ corresponds to (the equivalence class of) a Morita equivalence in $\mathbf{2 G p d}$.

In particular, the category $\mathbf{2} \mathbf{G} \mathbf{p d} \mathbf{d}_{\mathcal{M}}$ is the 1-category obtained from the bicategory $\mathbf{2} \mathbf{G p d}\left[\mathcal{M}^{-1}\right]$ (by identifying all 1 -morphisms that are connected by 2 -morphisms).

Lemma 2.17/3) implies that any generalized morphism can be represented by a chain of length 2 :

Lemma 2.18. Any generalized morphism between two Lie 2-groupoids $\boldsymbol{\Gamma}$ and $\boldsymbol{\Delta}$ is equivalent to a diagram

$$
\boldsymbol{\Gamma} \stackrel{\phi}{\sim} \boldsymbol{E} \stackrel{f}{\rightarrow} \boldsymbol{\Delta}
$$

in the category $\mathbf{2 G p d}$ in which $\phi$ is a hypercover (i.e. $\phi \in \mathcal{M}$ ). 
Remark 2.19. There is a bijection between maps of (representable) differentiable 2-stacks and equivalence classes of generalized morphisms of Lie 2-groupoids up to Morita equivalences.

\subsection{Lie 2-group bundles}

In this section, we give a definition of Lie 2-group bundles of a global nature and formulated in terms of generalized morphisms of Lie 2-groupoids.

Definition 2.20. A principal (Lie 2-group) $[G \rightarrow H]$-bundle over a Lie groupoid $\Gamma_{1} \rightrightarrows \Gamma_{0}$ is a generalized morphism $\mathfrak{B}$ from $\Gamma_{1} \rightrightarrows \Gamma_{0}$ (seen as a Lie 2-groupoid) to the Lie 2-group $[G \rightarrow H]$ associated to the crossed module $(G \rightarrow H)$.

In particular, a principal $[G \rightarrow \operatorname{Aut}(G)]$-bundle over a groupoid $\Gamma_{1} \rightrightarrows \Gamma_{0}$ is a generalized morphism from $\Gamma_{1} \rightrightarrows \Gamma_{0}$ (seen as a Lie 2-groupoid) to the Lie 2-group $[G \rightarrow \operatorname{Aut}(G)]$.

Two principal $[G \rightarrow H]$-bundles $\mathfrak{B}$ and $\mathfrak{B}^{\prime}$ over the Lie groupoid $\Gamma_{1} \rightrightarrows \Gamma_{0}$ are said to be isomorphic if, and only if, these two generalized morphisms are equivalent.

A $[G \rightarrow H]$-bundle over a manifold $M$ is a (2-group) $[G \rightarrow H]$-bundle over the Lie groupoid $M \rightrightarrows M$.

Let $\mathfrak{B}$ be a $[G \rightarrow H]$-bundle over a Lie groupoid $\Gamma_{1} \rightrightarrows \Gamma_{0}$. If $\Gamma_{1}^{\prime} \rightrightarrows \Gamma_{0}^{\prime}$ and $\left[G^{\prime} \rightarrow H^{\prime}\right]$ are Morita equivalent to $\Gamma_{1} \rightrightarrows \Gamma_{0}$ and $[G \rightarrow H]$ respectively, then the composition

$$
\left(\Gamma_{1}^{\prime} \rightrightarrows \Gamma_{0}^{\prime}\right) \leadsto\left(\Gamma_{1} \rightrightarrows \Gamma_{0}\right) \stackrel{\mathfrak{B}}{\rightsquigarrow}[G \rightarrow H] \leadsto\left[G^{\prime} \rightarrow H^{\prime}\right]
$$

defines a principal $\left[G^{\prime} \rightarrow H^{\prime}\right]$-bundle over $\Gamma_{1}^{\prime} \rightrightarrows \Gamma_{0}^{\prime}$ denoted $\mathfrak{B}$ by abuse of notation. Here the left and right squig arrows are the Morita equivalences seen as invertible generalized morphisms as in Example 2.15.

Definition 2.21. A principal (Lie 2-group) $[G \rightarrow H]$-bundle $\mathfrak{B}$ over a Lie groupoid $\Gamma_{1} \rightrightarrows \Gamma_{0}$ and a principal (Lie 2-group) $\left[G^{\prime} \rightarrow H^{\prime}\right]$-bundle $\mathfrak{B}^{\prime}$ over a Lie groupoid $\Gamma_{1}^{\prime} \rightrightarrows \Gamma_{0}^{\prime}$ are said to be Morita equivalent if, and only if, $\Gamma_{1}^{\prime} \rightrightarrows \Gamma_{0}^{\prime}$ is Morita equivalent to $\Gamma_{1} \rightrightarrows \Gamma_{0},\left[G^{\prime} \rightarrow H^{\prime}\right]$ is Morita equivalent to $[G \rightarrow H]$, and $\mathfrak{B}$ (viewed as a generalized morphism $\left(\Gamma_{1}^{\prime} \rightrightarrows \Gamma_{0}^{\prime}\right) \rightsquigarrow\left[G^{\prime} \rightarrow\right.$ $\left.H^{\prime}\right]$ ) and $\mathfrak{B}^{\prime}$ are equivalent generalized morphisms. 
Remark 2.22. When the groupoid is just a manifold, our definition is equivalent to the usual definition of Lie 2-group bundles in [3, 6, 50, 58] as suggested by Examples 2.23 and 2.24 below.

Furthermore our notion of isomorphism of principal 2-group bundles over a fixed manifold agrees with the one of [58] and is in fact a particular case of the one described in [59, see Remark 2.26.

Example 2.23. Let $P \stackrel{\pi}{\rightarrow} M$ be a principal $H$-bundle. Then the diagram

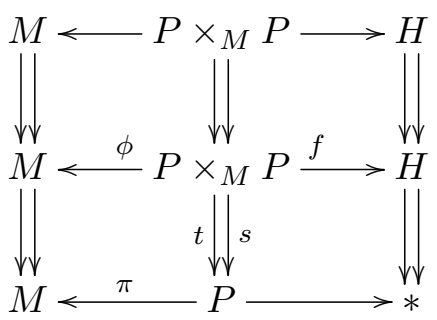

where $s(x, y)=x, t(x, y)=y, \pi(x)=\phi(x, y)=\pi(y)$ and $x \cdot f(x, y)=y$, defines a generalized morphism from the manifold $M$ to the 2-group $[1 \rightarrow H]$. Hence, it is a 2-group bundle over $M$. Note that a principal $H$-bundle $P$ over $M$ is Morita equivalent (as a 2-group bundle) to a principal $H^{\prime}$-bundle $P^{\prime}$ over $M^{\prime}$ if, and only if, $H$ and $M$ are isomorphic to $H^{\prime}$ and $M^{\prime}$ respectively and $P$ and $P^{\prime}$ are isomorphic principal bundles.

Example 2.24. Let $M$ be a smooth manifold and $G$ be a (non-abelian) Lie group. A non abelian 2-cocycle [18, 19, 26, 43] on $M$ with values in $G$ relative to an open covering $\left\{U_{i}\right\}_{i \in I}$ of $M$ is a collection of smooth maps

$$
\lambda_{i j}: U_{i j} \rightarrow \operatorname{Aut}(G) \quad \text { and } \quad g_{i j k}: U_{i j k} \rightarrow G
$$

satisfying the following relations:

$$
\begin{aligned}
& \lambda_{i j} \circ \lambda_{j k}=\mathbf{A d}_{g_{i j k}} \circ \lambda_{i k} \\
& g_{i j l} g_{j k l}=g_{i k l} \lambda_{k l}^{-1}\left(g_{i j k}\right) .
\end{aligned}
$$

Such a non-abelian 2-cocycle defines a $[G \rightarrow \operatorname{Aut}(G)]$-bundle over the manifold $M$; for it can be seen as the generalized morphism 


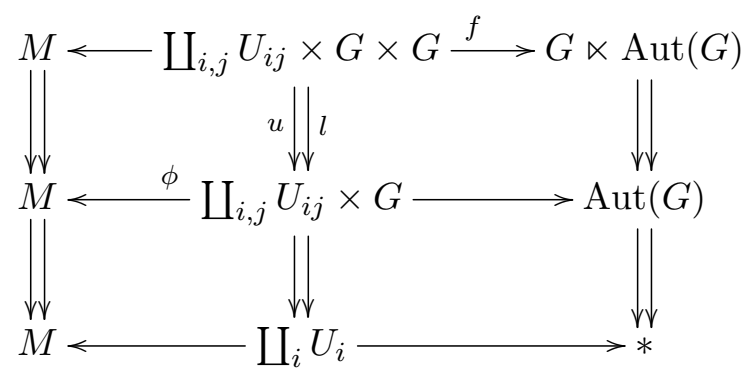

between the manifold $M$ and the 2-group $[G \rightarrow \operatorname{Aut}(G)]$. Here

$$
\begin{array}{ll}
l\left(x_{i j}, g_{1}, g_{2}\right)=\left(x_{i j}, g_{1}\right) & \phi\left(x_{i j}, g\right)=x \\
u\left(x_{i j}, g_{1}, g_{2}\right)=\left(x_{i j}, g_{2}\right) & f\left(x_{i j}, g_{1}, g_{2}\right)=\left(g_{2} g_{1}^{-1}, \mathbf{A d}_{g_{1}} \circ \lambda_{i j}(x)\right)
\end{array}
$$

where $x_{i j}$ denotes a point $x \in M$ seen as a point of the open subset $U_{i j}=$ $U_{i} \cap U_{j}, x_{i}$ the point $x \in M$ seen as a point of the open subset $U_{i}$, and $g, g_{1}, g_{2}$ arbitrary elements of $G$. The horizontal and vertical multiplications are given by

$$
\begin{aligned}
& \left(x_{i j}, g_{1}\right) *\left(x_{j k}, g_{2}\right)=\left(x_{i k}, g_{i j k} \lambda_{j k}^{-1}\left(g_{1}\right) g_{2}\right), \\
& \left(x_{i j}, g_{1}, g_{2}\right) \star\left(x_{i j}, g_{2}, g_{3}\right)=\left(x_{i j}, g_{1}, g_{3}\right) .
\end{aligned}
$$

Example 2.25. Let $\left\{U_{i}\right\}_{i \in I}$ be an open covering of a smooth manifold $M$. A family of smooth maps $g_{i j k}: U_{i j k} \rightarrow S^{1}$ defines a Lie groupoid structure on $\coprod_{i, j} U_{i j} \times S^{1} \rightrightarrows \coprod_{i} U_{i}$ with multiplication

$$
\left(x_{i j}, e^{i \varphi}\right) \cdot\left(x_{j k}, e^{i \psi}\right)=\left(x_{i k}, g_{i j k} e^{i(\varphi+\psi)}\right)
$$

if, and only if, $g_{i j k}$ is a Čech 2-cocycle. In that case, we get the generalized morphism of 2-groupoids

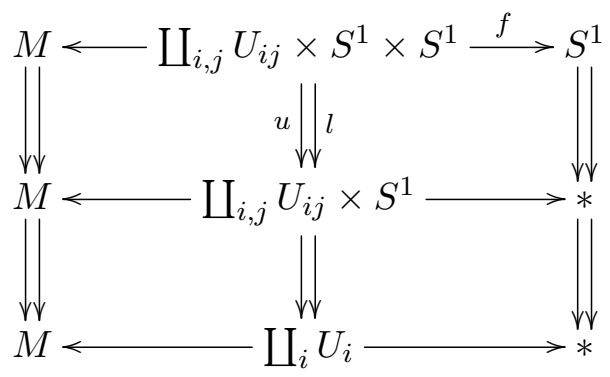

with $f\left(x_{i j}, e^{i \varphi}, e^{i \psi}\right)=e^{i(\psi-\varphi)}$. It defines an $\left[S^{1} \rightarrow *\right]$-bundle over $M$. 
Remark 2.26. Our definition of principal 2-groups bundles over a stack also agrees with one which was introduced more recently by Wolfson in [59]; more precisely our definition agrees with a special case of principal bundle over a simplicial Lie group in loc. cit. when the simplicial Lie group is a strict Lie 2-group (viewed as a special kind of simplicial Lie groups as in [59, $\S 6])$. Indeed, given a generalized morphism $\left(\Gamma_{1} \rightrightarrows \Gamma_{0}\right) \stackrel{\mathfrak{B}}{\rightsquigarrow}[G \rightarrow H]$, we get a strict Lie 2-groupoid map $\boldsymbol{U} \rightarrow[G \rightarrow H]$, for some hypercover $\stackrel{\boldsymbol{U}}{\longrightarrow} \boldsymbol{\Gamma}$, which in turn yields a map between the associated simplicial manifolds. Pulling back the universal bundle $W([G \rightarrow H])$ of [59, Definition 6.2] along this map yields a twisted Cartesian product $\boldsymbol{U} \times[G \rightarrow H] W([G \rightarrow H]) \rightarrow \boldsymbol{U}$ over (the simplicial manifold associated to) $\boldsymbol{U}$ (which is a stack by construction). Since $\boldsymbol{U} \stackrel{\phi}{\longrightarrow} \boldsymbol{\Gamma}$ is a hypercover, this is precisely the data of a local 2-bundle which is principal with respect to (the simplicial Lie group associated to) $[G \rightarrow H]$ in the sense of [59, §5].

Furthermore, by choosing a common refinement of two hypercovers, an isomorphism of principal 2-group bundles in the sense of Definition 2.21 yields an equivalence of local 2-bundles (as twisted cartesian product and as stacks), since in both cases it boils down to being a collection of equivalences of local objects of the form $\boldsymbol{E} \cong \boldsymbol{U} \times[G \rightarrow H]$.

Example 2.27. Principal 2-group bundles arise whenever one studies group actions on stacks, which, in general are only weak actions. For instance, there is a canonical (but subtle) weak action of $S^{1}$ on the inertia stack of any differentiable stack giving rise to a canonical principal bundle, which shall be detailed elsewhere. For the moment we just explain briefly how it can be defined in terms of Lie 2-group(oid)s. Let $\Gamma: \Gamma_{1} \rightrightarrows \Gamma_{0}$ be a Lie groupoid. Its inertia groupoid $3 \Lambda: S_{\Gamma} \times_{\Gamma_{0}} \Gamma_{1} \rightrightarrows S_{\Gamma}$ (where $S_{\Gamma}=\left\{\gamma \in \Gamma_{1}\right.$ s.t. $d(\gamma)=$ $s(\gamma)\}$ is the space of loops) has a canonical action of the group stack associated to the 2 -group $[\mathbb{Z} \rightarrow 1]$. This action is given, for $(\gamma, g) \in S_{\Gamma} \times \Gamma_{1}$ and $n \in \mathbb{Z}$ by $(\gamma, g) \cdot n:=\left(\gamma, \gamma^{n} \cdot g\right)$. Hence it also inherits an action of the group stack $S^{1} \cong[\mathbb{Z} \rightarrow \mathbb{R}]$ (induced by the canonical map $\mathbb{R} \rightarrow 0$ ). One shall note that this action is almost never represented by a strict action of the group $S^{1}$ on the inertia groupoid but really by an action of the 2-group $[\mathbb{Z} \rightarrow \mathbb{R}]$. Assume the inertia groupoid is a Lie groupoid - which is true if $\boldsymbol{\Gamma}$ is étale and proper. It follows from [24, Theorem 0.2] that the quotient of the stack represented by $\Lambda \Gamma$ by the (group stack represented by) $S^{1} \cong[\mathbb{Z} \rightarrow \mathbb{R}]$ is a

\footnotetext{
${ }^{3}$ The inertia groupoid of a groupoid $\Gamma_{1} \rightrightarrows \Gamma_{0}$ is a groupoid representing the inertia stack of the quotient stack $\left[\Gamma_{0} / \Gamma_{1}\right]$. See [7] for details on inertia groupoids and stacks.
} 
differentiable stack and further a $[\mathbb{Z} \rightarrow \mathbb{R}]$-principal bundle. Indeed this quotient stack can be presented by a Lie groupoid $\tilde{\tilde{\Gamma}}$ which is Morita equivalent to the Lie 2-groupoid $\tilde{\tilde{\Gamma}}: S_{\Gamma} \times{ }_{\Gamma_{0}} \Gamma_{1} \times \mathbb{Z} \times \mathbb{R} \rightrightarrows S_{\Gamma} \times_{\Gamma_{0}} \Gamma_{1} \times \mathbb{R} \rightrightarrows S_{\Gamma}$. The horizontal multiplications are given by the product of the Lie (2-)groupoids structures of $\Gamma$ and $[\mathbb{Z} \rightarrow \mathbb{R}]$ while vertical mutiplication is induced by the action $\underset{\tilde{\Gamma}}{\mathbb{Z}}$ on the inertia groupoid described above. The canonical projection $\tilde{\tilde{\Gamma}} \rightarrow[\mathbb{Z} \underset{\tilde{\tilde{\Gamma}}}{\rightarrow}]=\mathbb{Z} \times \mathbb{R} \rightrightarrows \mathbb{R} \rightrightarrows 1$ gives right to the generalized morphism $\mathcal{B}: \tilde{\boldsymbol{\Gamma}} \stackrel{\tilde{\tilde{\Gamma}}}{\leftarrow} \rightarrow[\mathbb{Z} \rightarrow \mathbb{R}]$ defining the bundle structure.

Remark 2.28. There is a nerve functor from Lie 2-groupoids to simplicial spaces generalizing the nerve for Lie 1-groupoids. For instance, see [16, 51, 55. and Section 4.1 below. Composing it with the (fat) realization functor, we obtain the classifying space functor $\boldsymbol{\Gamma} \mapsto B \Gamma$ from Lie 2-groupoids to topological spaces. Since the realization of a Morita morphism (i.e. hypercover) is a homotopy equivalence, a generalized morphism $\Gamma \stackrel{F}{\sim} \boldsymbol{\Delta}$ induces a map $B \boldsymbol{\Gamma} \stackrel{B F}{\longrightarrow} B \boldsymbol{\Delta}$ in the homotopy category of topological spaces. In particular, a $[G \rightarrow H]$-group bundle over a manifold induces a map $M \rightarrow B[G \rightarrow H]$ in the homotopy category. This is the topological side of generalized morphisms and 2-group bundles. In fact, using standard arguments on homotopy for manifolds, it should be possible to prove that $[G \rightarrow H]$-group bundles over $\boldsymbol{\Gamma}$ (up to Morita equivalences) are in bijection with homotopy classes of maps $B \Gamma \rightarrow B[G \rightarrow H]$.

\section{Groupoid $G$-extensions}

We fix a Lie group $G$. We recall the following definition (see [33])

Definition 3.1. A Lie groupoid G-extension is a short exact sequence of Lie groupoids over the identity map on the unit space $M$

$$
1 \rightarrow M \times G \stackrel{i}{\rightarrow} \tilde{\Gamma} \stackrel{\phi}{\rightarrow} \Gamma \rightarrow 1
$$

Here both $\Gamma$ and $\tilde{\Gamma}$ are Lie groupoids over $M$ and $M \times G \rightrightarrows M$ is a (trivial) bundle of groups.

The map $\phi$ being a map over the identity map on the unit space $M$ means that both $\tilde{\Gamma}$ and $\Gamma$ have $M$ for unit space and that the restriction of 
$\phi$ to $M$ is the identity on $M$ :

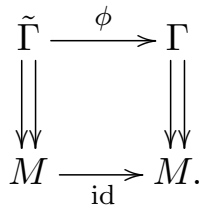

In the sequel, an extension like (6) will be denoted $\tilde{\Gamma} \stackrel{\phi}{\rightarrow} \Gamma \rightrightarrows M$ and we will write $g_{m}$ instead of $i(m, g)$.

Lie groupoid $G$-extensions can be interpreted in terms of crossed modules as follows.

Proposition 3.2. The morphism of groupoids $\tilde{\Gamma} \stackrel{\phi}{\rightarrow} \Gamma \rightrightarrows M$ is a groupoid G-extension if, and only if, $(M \times G \stackrel{i}{\hookrightarrow} \tilde{\Gamma})$ is a crossed module of groupoids with quotient groupoid $\tilde{\Gamma} / i(M \times G)$ isomorphic to $\Gamma$.

Proposition 3.2 follows easily from Remark 3.7 and Lemma 3.9 below.

Definition 3.3 ([33]). A Morita morphism between Lie groupoid G-extensions is a homomorphism of Lie groupoid $G$-extensions

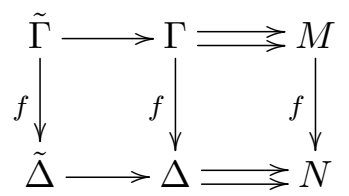

such that $M \stackrel{f}{\rightarrow} N$ is a surjective submersion and

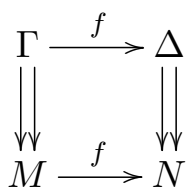

and

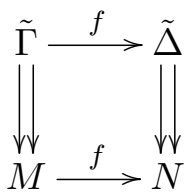

are Morita morphisms ${ }^{4}$ of 1-groupoids.

As in the Lie 2-groupoid case, the Morita morphisms of Lie groupoid extensions form a left multiplicative system in the category of Lie groupoid extensions and homomorphisms of Lie groupoid extensions. Hence, one can

\footnotetext{
${ }^{4}$ Weak equivalences in [44] and hypercovers in [59, 60].
} 
localize this category by its Morita morphisms. Two Lie groupoid extensions are Morita equivalent if they are isomorphic in the localized category. (As in the Lie 2-groupoids case, there is a notion of generalized morphisms for Lie groupoid extensions. In that language, a Morita equivalence is an invertible generalized morphism.)

Here is our first main theorem.

Theorem 3.4. There exists a bijection between the Morita equivalence classes of Lie groupoid G-extensions and the Morita equivalence classes of $[G \rightarrow \operatorname{Aut}(G)]$-bundles over Lie groupoids.

Remark 3.5. The above theorem can be regarded as a geometric version of a theorem of Breen [12], which states that $H^{2}(\mathfrak{X}, G)$ is isomorphic to $H^{1}(\mathfrak{X},(G \rightarrow \operatorname{Aut}(G)))$.

The proof of Theorem 3.4 is the object of the next two sections.

\subsection{From groupoid $G$-extensions to $[G \rightarrow \operatorname{Aut}(G)]$-bundles}

Given a Lie groupoid G-extension $\tilde{\Gamma} \stackrel{\phi}{\longrightarrow} \Gamma \underset{b}{\stackrel{a}{\rightleftarrows}} M$, one can define a Lie 2-groupoid $\tilde{\Gamma} \times_{\Gamma} \tilde{\Gamma} \underset{u}{\stackrel{l}{\rightleftarrows}} \tilde{\Gamma} \underset{t}{\stackrel{s}{\rightleftarrows}} M$, where

$$
\begin{gathered}
\tilde{\Gamma} \times_{\Gamma} \tilde{\Gamma}=\left\{\left(\tilde{\gamma}_{1}, \tilde{\gamma}_{2}\right) \in \tilde{\Gamma} \times \tilde{\Gamma} \mid \phi\left(\tilde{\gamma}_{1}\right)=\phi\left(\tilde{\gamma}_{2}\right)\right\} \\
l\left(\tilde{\gamma}_{1}, \tilde{\gamma}_{2}\right)=\tilde{\gamma}_{2} \quad u\left(\tilde{\gamma}_{1}, \tilde{\gamma}_{2}\right)=\tilde{\gamma}_{1} \\
s(\tilde{\gamma})=a(\phi(\tilde{\gamma})) \quad t(\tilde{\gamma})=b(\phi(\tilde{\gamma})) \\
\left(\tilde{\gamma}_{1}, \tilde{\gamma}_{2}\right) \star\left(\tilde{\gamma}_{2}, \tilde{\gamma}_{3}\right)=\left(\tilde{\gamma}_{1}, \tilde{\gamma}_{3}\right) \\
\left(\tilde{\gamma}_{1}, \tilde{\gamma}_{2}\right) *\left(\tilde{\delta}_{1}, \tilde{\delta}_{2}\right)=\left(\tilde{\gamma}_{1} \cdot \tilde{\delta}_{1}, \tilde{\gamma}_{2} \cdot \tilde{\delta}_{2}\right) .
\end{gathered}
$$

Here $\cdot$ stands for the multiplication in $\tilde{\Gamma} \rightrightarrows M$.

The groupoid homomorphism $\phi$ naturally induces a Morita morphism (i.e. hypercover) of 2-groupoids:

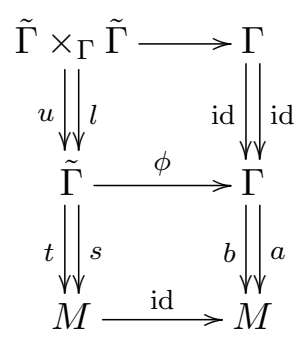


where the 2-groupoid $\Gamma \underset{\text { id }}{\stackrel{\text { id }}{\rightleftarrows}} \Gamma \underset{b}{\stackrel{a}{\rightleftarrows}} M$ is simply the 1 -groupoid $\Gamma \underset{b}{\stackrel{a}{\rightleftarrows}} M$ seen as a 2-groupoid in the trivial way.

Consider the map $\tilde{\Gamma} \rightarrow \operatorname{Aut}(G): \tilde{\gamma} \mapsto \operatorname{Ad}_{\tilde{\gamma}}$ defined by $\left(\operatorname{Ad}_{\tilde{\gamma}} g\right)_{t(\tilde{\gamma})}=\tilde{\gamma} \cdot$ $g_{s(\tilde{\gamma})} \cdot \tilde{\gamma}^{-1}$. It gives a morphism of Lie groupoids

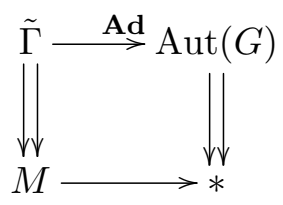

which, together with the map

$$
\tilde{\Gamma} \times_{\Gamma} \tilde{\Gamma} \rightarrow G \ltimes \operatorname{Aut}(G):\left(\tilde{\gamma}_{1}, \tilde{\gamma}_{2}\right) \mapsto\left(g, \operatorname{Ad}_{\tilde{\gamma}_{2}}\right),
$$

where $\tilde{\gamma}_{1} \tilde{\gamma}_{2}^{-1}=g_{t\left(\tilde{\gamma}_{1}\right)}$, defines a homomorphism of Lie 2-groupoids

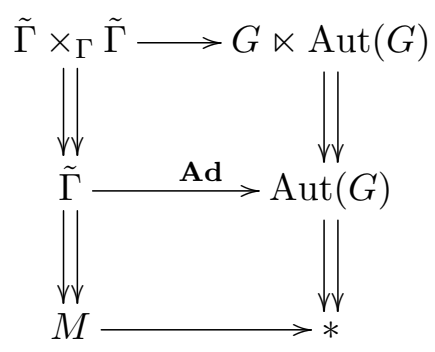

Remark 3.6. Note that the induced map

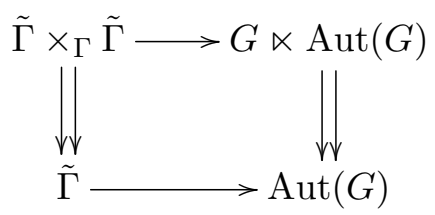

is a fully faithful functor.

Remark 3.7. In terms of crossed modules, the above discussion goes as follows. The extension $1 \rightarrow M \times G \stackrel{i}{\rightarrow} \tilde{\Gamma} \stackrel{\phi}{\rightarrow} \Gamma \rightarrow 1$ leads to an action of $\tilde{\Gamma}$ on the groupoid $M \times G \rightrightarrows M$ by conjugation, i.e. via the map $\tilde{\gamma} \mapsto \mathbf{A d}_{\tilde{\gamma}}$. Then $\tilde{\Gamma} \times_{\Gamma} \tilde{\Gamma} \underset{u}{\stackrel{l}{\rightleftarrows}} \tilde{\Gamma} \underset{t}{\stackrel{s}{\rightleftarrows}} M$ is the Lie 2-groupoid corresponding to the crossed module $(M \times G \stackrel{i}{\rightarrow} \tilde{\Gamma})$. The projection onto the first factor $M \times G \rightarrow M$ and 
the morphism $\phi: \tilde{\Gamma} \rightarrow \Gamma$ induce the Morita equivalence of crossed modules $(M \times G \rightarrow \tilde{\Gamma}) \rightarrow(M \rightarrow \Gamma)$ corresponding to the map (7). Moreover, the map Ad : $\tilde{\Gamma} \rightarrow \operatorname{Aut}(G)$ yields the map of crossed modules $(M \times G \stackrel{i}{\rightarrow} \tilde{\Gamma}) \stackrel{\left(\mathrm{pr}_{2}, \text { Ad }\right)}{\longrightarrow}$ $(G \rightarrow \operatorname{Aut}(G))$ corresponding to the morphism of Lie 2-groupoids (9).

Proposition 3.8. $\quad 1)$ A Lie groupoid $G$-extension $\tilde{\Gamma} \rightarrow \Gamma \rightrightarrows M$ induces a principal $[G \rightarrow \operatorname{Aut}(G)]$-bundle over $\Gamma \rightrightarrows M$, which can be described explicitly by the following generalized morphism:

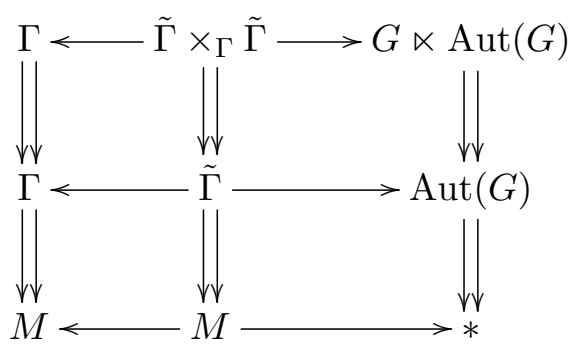

2) If $\tilde{\Gamma} \rightarrow \Gamma \rightrightarrows M$ and $\tilde{\Delta} \rightarrow \Delta \rightrightarrows N$ are Morita equivalent $G$-extensions, then the corresponding 2-group bundles are Morita equivalent.

Proof. Claim 1) follows from the above discussion. Suppose given a Morita morphism of $G$-extensions

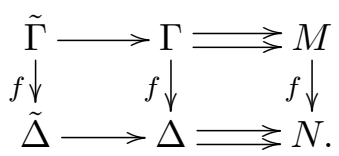

Since $f$ commutes with the $\tilde{\Gamma}$ and $\tilde{\Delta}$-actions on $G$, there is a commutative diagram

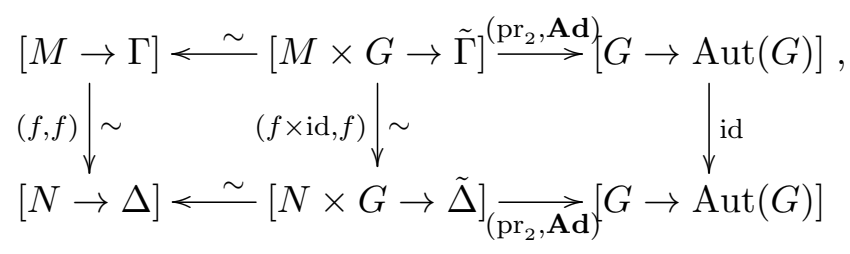

where $p_{2}$ denotes the canonical projection on the second component. Now, Claim 2 follows from Example 2.14. 


\subsection{From $[G \rightarrow \operatorname{Aut}(G)]$-bundles to groupoid $G$-extensions}

In this section, we show how to reverse the procedure. Starting from a $[G \rightarrow$ $\operatorname{Aut}(G)$ ]-bundle, we recover a groupoid $G$-extension.

For future reference, we state the following technical result without proof.

Lemma 3.9. Let

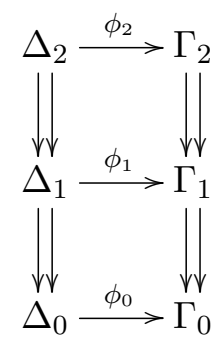

be a hypercover of Lie 2-groupoids. And let

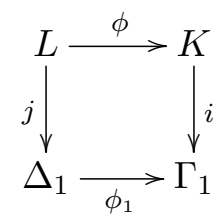

be the induced map of crossed modules. Then $\phi$ maps $j^{-1}\left(1_{m}\right)$ onto $i^{-1}\left(1_{\phi(m)}\right)$ bijectively (for every $m \in \Delta_{0}$ ) and induces a functor from the groupoid $\frac{\Delta_{1}}{j(L)}$ to the groupoid $\frac{\Gamma_{1}}{i(K)}$, which is fully faithful and surjective on the objects ${ }^{5}$

Now, given a $[G \rightarrow \operatorname{Aut}(G)]$-bundle over a Lie groupoid $\Gamma \rightrightarrows \Gamma_{0}$, we proceed with the construction of a Lie groupoid $G$-extension. Suppose the $[G \rightarrow \operatorname{Aut}(G)]$-bundle is given by the generalized morphism of 2-groupoids

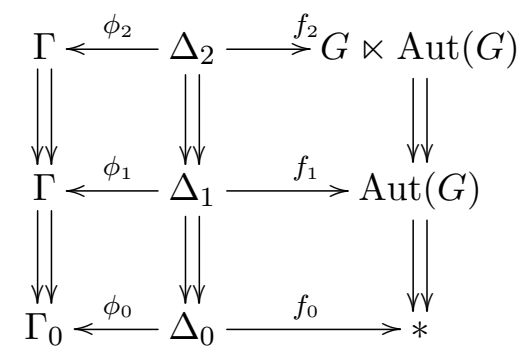

\footnotetext{
${ }^{5}$ The crossed modules $\left[1 \rightarrow \frac{\Delta_{1}}{j(L)}\right]$ and $\left[1 \rightarrow \frac{\Gamma_{1}}{i(K)}\right]$ are the 'cokernels' of the crossed modules $\left[L \stackrel{j}{\rightarrow} \Delta_{1}\right]$ and $\left[K \stackrel{i}{\rightarrow} \Gamma_{1}\right]$ respectively.
} 
and let

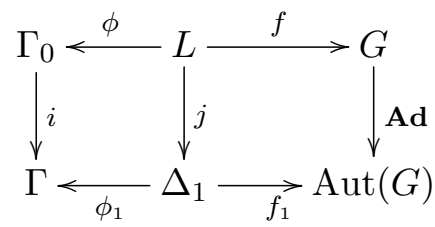

be the induced generalized morphism of crossed modules. Hence

$$
L=\left\{\alpha \in \Delta_{2} \mid u(\alpha)=1_{x} \text { for some } x \in \Delta_{0}\right\}
$$

and $j: L \rightarrow \Delta_{1}$ is the restriction of the structure map $l: \Delta_{2} \rightarrow \Delta_{1}$ to $L$. Since $\phi$ is a hypercover and $\Gamma_{0} \stackrel{i}{\rightarrow} \Gamma$ is an injection, by Lemma $3.9, L \stackrel{j}{\rightarrow} \Delta_{1}$ is also injective and

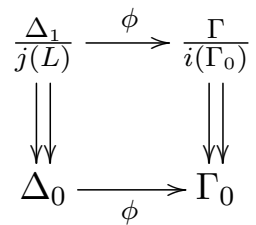

is a fully faithful functor. Since $\frac{\Gamma}{i\left(\Gamma_{0}\right)}$ is diffeomorphic to $\Gamma$, the groupoid $\frac{\Delta_{1}}{j(L)} \rightrightarrows \Delta_{0}$ is the pullback of $\Gamma \rightrightarrows \Gamma_{0}$ through the surjective submersion $\Delta_{0} \stackrel{\phi}{\rightarrow} \Gamma_{0}$. Therefore $\frac{\Delta_{1}}{j(L)}=\Delta_{0} \times_{\phi, \Gamma_{0}, s} \Gamma \times_{t, \Gamma_{0}, \phi} \Delta_{0}$ is a smooth manifold.

Consider the groupoid structure on $\Delta_{1} \times G \rightrightarrows \Delta_{0}$ with source $s(\delta, g)=$ $s(\delta)$, target $t(\delta, g)=t(\delta)$, mutliplication

$$
\left(\delta_{1}, g_{1}\right) \cdot\left(\delta_{2}, g_{2}\right)=\left(\delta_{1} \delta_{2}, f_{1}\left(\delta_{2}^{-1}\right)\left[g_{1}\right] \cdot g_{2}\right)
$$

and inverse $(\delta, g)^{-1}=\left(\delta^{-1}, f_{1}(\delta)\left[g^{-1}\right]\right)$ for all $\delta, \delta_{1}, \delta_{2} \in \Delta_{1}$ and $g, g_{1}, g_{2} \in G$.

The map $\mathcal{H}: L \rightarrow \Delta_{1} \times G$ defined by $\mathcal{H}(\alpha)=\left(j(\alpha), f\left(\alpha^{-1}\right)\right)$ is a morphism of groupoids from $L \rightrightarrows \Delta_{0}$ to $\Delta_{1} \times G \rightrightarrows \Delta_{0}$. One checks that

$$
\begin{aligned}
(\delta, g) \cdot\left(j(\alpha), f\left(\alpha^{-1}\right)\right) & =\left(j\left(\delta * \alpha * \delta^{-1}\right), f\left(\delta * \alpha * \delta^{-1}\right)^{-1}\right) \cdot(\delta, g) \\
& =\left(\delta \cdot j(\alpha), f\left(\alpha^{-1}\right) \cdot g\right)
\end{aligned}
$$

for all $\delta \in \Delta_{1}, g \in G$, and $\alpha \in L$. Thus the image of $L$ under $\mathcal{H}$ is a normal subgroupoid of $\Delta_{1} \times G \rightrightarrows \Delta_{0}$. Since $j: L \rightarrow \Delta_{1}$ is injective, the action of $L$ on $\Delta_{1} \times G$ by multiplication from the right

$$
(\delta, g) \bullet \alpha=(\delta, g) \cdot\left(j(\alpha), f\left(\alpha^{-1}\right)\right)=\left(\delta \cdot j(\alpha), f\left(\alpha^{-1}\right) \cdot g\right)
$$

is free and its orbit space $\left(\Delta_{1} \times G\right) / \mathcal{H}(L)$ is a smooth manifold. 
Now consider the groupoid $G$-extension $\Delta_{1} \times G \rightarrow \Delta_{1} \rightrightarrows \Delta_{0}$. The morphism of groupoids $\Delta_{1} \times G \ni(\delta, g) \mapsto \delta \in \Delta_{1}$ intertwines the right action of $L$ on $\Delta_{1} \times G$ with the right action $\delta \bullet \alpha=\delta \cdot j(\alpha)$ of $L$ on $\Delta_{1}$, whose orbit space is the smooth manifold $\frac{\Delta_{1}}{j(L)}$. Therefore, passing to quotients, we obtain the $G$-extension of groupoids

$$
\left(\Delta_{1} \times G\right) / \mathcal{H}(L) \rightarrow \Delta_{1} / j(L) \rightrightarrows \Delta_{0}
$$

Note that the corresponding crossed module is

$$
\left(\Delta_{0} \times G \rightarrow\left(\Delta_{1} \times G\right) / \mathcal{H}(L)\right)
$$

Proposition 3.10. 1) Every $[G \rightarrow \operatorname{Aut}(G)]$-bundle over a Lie groupoid $\Gamma \rightrightarrows \Gamma_{0}$ induces a Lie groupoid $G$-extension.

2) Morita equivalent $[G \rightarrow \operatorname{Aut}(G)]$-bundles induce Morita equivalent extensions.

Proof. 1) As was outlined above, a bundle

$$
\left[\Gamma_{0} \rightarrow \Gamma\right] \stackrel{\phi}{\leftarrow} \boldsymbol{\Delta} \stackrel{f}{\rightarrow}[G \rightarrow \operatorname{Aut}(G)]
$$

determines a $G$-extension

$$
\left(\Delta_{1} \times G\right) / \mathcal{H}(L) \rightarrow \Delta_{1} / j(L) \rightrightarrows \Delta_{0},
$$

where $j: L \rightarrow \Delta_{1}$ is the restriction of the structure map $l: \Delta_{2} \rightarrow \Delta_{1}$ to

$$
L=\left\{\alpha \in \Delta_{2} \mid u(\alpha)=1_{x} \text { for some } x \in \Delta_{0}\right\}
$$

and $\mathcal{H}: L \rightarrow \Delta_{1} \times G$ is the morphism of groupoids from $L \rightrightarrows \Delta_{0}$ to $\Delta_{1} \times$ $G \rightrightarrows \Delta_{0}$ defined by $\mathcal{H}(\alpha)=\left(j(\alpha), f\left(\alpha^{-1}\right)\right)$.

2. It is sufficient to check that for any diagram

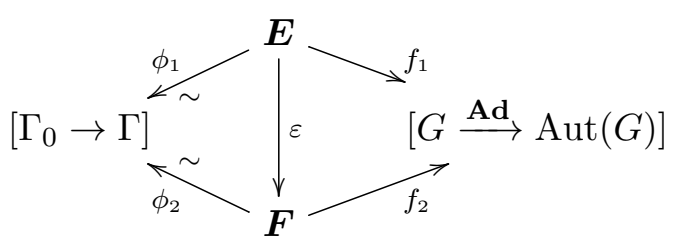

commuting up to natural 2-equivalences, the $G$-extensions corresponding to the lower and upper generalized morphisms are Morita equivalent. Since 
$\phi_{1}, \phi_{2}$ are Morita equivalences, $\varepsilon$ is also a Morita equivalence. Therefore, by Lemma 2.18, we can assume that $\varepsilon$ is a hypercover. Then, denoting by $\left(K \rightarrow E_{1}\right)$ and $\left(L \rightarrow F_{1}\right)$ the crossed modules corresponding to $\boldsymbol{E}$ and $\boldsymbol{F}$ respectively, the map $\varepsilon$ induces a commutative diagram

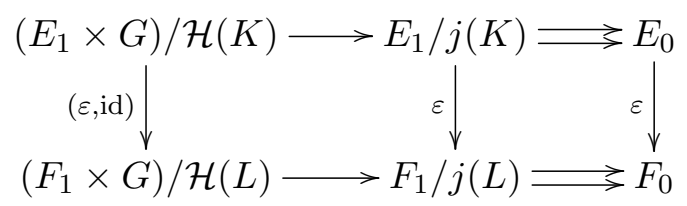

which is a Morita equivalence of extensions by Lemma 3.9 .

\subsection{Proof of Theorem 3.4}

It remains to prove that the constructions of Section 3.1 and Section 3.2 are inverse of each other.

Suppose that a $[G \rightarrow \operatorname{Aut}(G)]$-principal bundle $\mathfrak{B}$ over $\Gamma$ is given by the generalized morphism $\Gamma \stackrel{\phi}{\leftarrow} \Delta \stackrel{f}{\rightarrow}[G \rightarrow \operatorname{Aut}(G)]$. Let $\frac{\Delta_{1} \times G}{\mathcal{H}(L)} \rightarrow \frac{\Delta_{1}}{j(L)} \rightrightarrows \Delta_{0}$ be the induced $G$-principal extension as in Proposition 3.101 ). The corresponding crossed module is $\left(\Delta_{0} \times G \rightarrow \frac{\Delta_{1} \times G}{\mathcal{H}(L)}\right)$. We have the following commutative diagram of crossed modules:

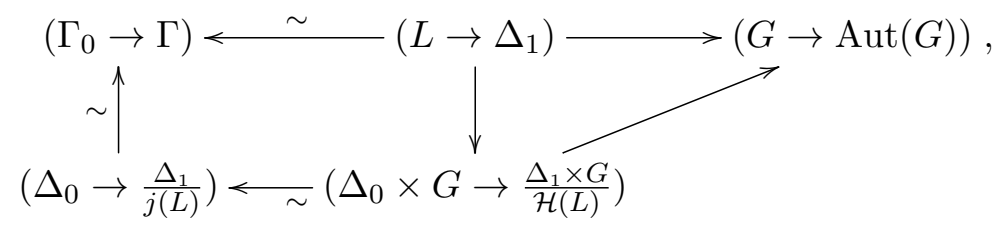

where $\left(L \rightarrow \Delta_{1}\right)$ is the crossed module corresponding to $\boldsymbol{\Delta}$. It follows that the generalized morphism

$$
\left[\Gamma_{0} \rightarrow \Gamma\right] \stackrel{\sim}{\leftarrow}\left[L \rightarrow \Delta_{1}\right] \rightarrow[G \rightarrow \operatorname{Aut}(G)]
$$

we started from is equivalent to the generalized morphism

$$
\left[\Gamma_{0} \rightarrow \Gamma\right] \stackrel{\sim}{\leftarrow}\left[\Delta_{0} \times G \rightarrow \frac{\Delta_{1} \times G}{\mathcal{H}(L)}\right] \rightarrow[G \rightarrow \operatorname{Aut}(G)]
$$

associated to the $G$-extension $\frac{\Delta_{1} \times G}{\mathcal{H}(L)} \rightarrow \frac{\Delta_{1}}{j(L)} \rightrightarrows \Delta_{0}$. Hence they represent the same $(G \rightarrow \operatorname{Aut}(G))$-bundle over $\Gamma \rightrightarrows \Gamma_{0}$. 
Reciprocally, if $\tilde{\Gamma} \rightarrow \Gamma \rightrightarrows M$ is a $G$-extension, then the associated principal $[G \rightarrow \operatorname{Aut}(G)]$-bundle is given by the generalized morphism

$$
[M \rightarrow \Gamma] \stackrel{\phi}{\leftarrow}[M \times G \rightarrow \tilde{\Gamma}] \stackrel{\left(\mathrm{pr}_{2}, \mathrm{Ad}\right)}{\longrightarrow}[G \rightarrow \operatorname{Aut}(G)]
$$

according to Remark 3.7. Direct inspection of the proof of Proposition 3.10 shows that the $G$-extension induced by the generalized morphism 10 is exactly $\tilde{\Gamma} \rightarrow \Gamma \rightrightarrows M$.

\section{Universal characteristic maps and Dixmier-Douady classes}

\subsection{Cohomology of Lie 2-groupoids}

To each Lie 2-groupoid $\Gamma_{2} \rightrightarrows \Gamma_{1} \rightrightarrows \Gamma_{0}$ is associated a simplicial manifold: its (geometric) nerve $N_{\bullet} \boldsymbol{\Gamma}$. It is the nerve of the underlying 2-category as defined by Street [55]. In particular, $N_{0} \boldsymbol{\Gamma}=\Gamma_{0}, N_{1} \boldsymbol{\Gamma}=\Gamma_{1}, N_{2} \boldsymbol{\Gamma}$ is a submanifold of $\Gamma_{2} \times \Gamma_{1} \times \Gamma_{1} \times \Gamma_{1}$ parameterizing the 2-arrows of $\Gamma_{2}$ fitting in a commutative triangle

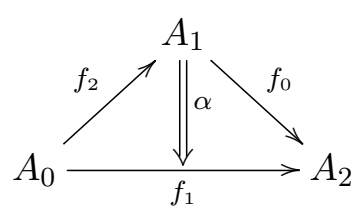

and $N_{3} \boldsymbol{\Gamma}$ is a submanifold of $\left(\Gamma_{2}\right)^{4} \times\left(\Gamma_{1}\right)^{6}$ parameterizing the commutative tetrahedra like

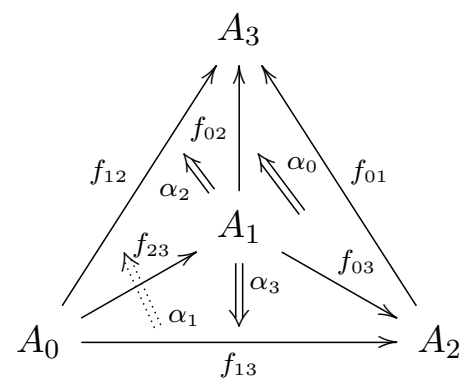

with faces given by elements of $N_{2} \Gamma$. By the commutativity of the tetrahedron $(12)$, we mean that $\left(\alpha_{3} * f_{01}\right) \star \alpha_{1}=\left(f_{23} * \alpha_{0}\right) \star \alpha_{2}$. For $p \geq 3, N_{p} \boldsymbol{\Gamma}$ is the manifold of all $p$-simplices such that each subsimplex of dimension 3 is 
a tetrahedron of the form (12) above [22, 55]. The nerve of a Lie groupoid considered as a Lie 2-groupoid is isomorphic to its usual (1-)nerve [51]. The nerve $N$. defines a functor from the category of Lie 2-groupoids to the category of simplicial manifolds. Taking the fat realization of the nerve defines a functor from Lie 2-groupoids to topological spaces.

The de Rham cohomology groups of a Lie 2-groupoid $\boldsymbol{\Gamma}$ are defined to be the total cohomology groups of the bicomplex $\left(\Omega^{\bullet}\left(N_{\bullet} \Gamma\right), d_{\mathrm{DR}}, \partial\right)$, where $d_{\mathrm{DR}}: \Omega^{p}\left(N_{q} \boldsymbol{\Gamma}\right) \rightarrow \Omega^{p+1}\left(N_{q} \boldsymbol{\Gamma}\right)$ is the de Rham differential and $\partial$ : $\Omega^{p}\left(N_{q} \boldsymbol{\Gamma}\right) \rightarrow \Omega^{p}\left(N_{q+1} \boldsymbol{\Gamma}\right)$ is defined by $\partial=(-1)^{p} \sum_{i=0}^{q+1}(-1)^{i} d_{i}^{*}$, where $d_{i}$ : $N_{q+1} \boldsymbol{\Gamma} \rightarrow N_{q} \boldsymbol{\Gamma}$ denotes the $i^{\text {th }}$ face map. We use the shorter notation $\Omega_{\text {tot }}(\boldsymbol{\Gamma})$ for the associated total complex. Hence $\Omega_{\text {tot }}^{n}(\boldsymbol{\Gamma})=\bigoplus_{p+q=n} \Omega^{p}\left(N_{q} \boldsymbol{\Gamma}\right)$ with (total) differential $d_{\mathrm{DR}}+\partial$. We denote the subspaces of cocycles and coboundaries by $Z_{\mathrm{DR}}^{\bullet}(\boldsymbol{\Gamma})$ and $B_{\mathrm{DR}}^{\bullet}(\boldsymbol{\Gamma})$ respectively, and the cohomology of $\boldsymbol{\Gamma}$ by $H \cdot(\boldsymbol{\Gamma})$.

The following Lemma is folklore (see [20] for a more general statement with respect to hypercovers).

Lemma 4.1. Let $F: \boldsymbol{\Gamma} \rightarrow \boldsymbol{\Delta}$ be a hypercover of Lie 2-groupoids. Then $F^{*}$ : $H^{\bullet}(\boldsymbol{\Delta}) \rightarrow H^{\bullet}(\boldsymbol{\Gamma})$ is an isomorphism.

Proof. It is well-known that a natural transformation between two 2-functors $f$ and $g$ from $\boldsymbol{\Gamma}$ to $\boldsymbol{\Delta}$ induces a simplicial homotopy between $f_{*}: N_{\bullet}(\boldsymbol{\Gamma}) \rightarrow$ $N_{\bullet}(\boldsymbol{\Delta})$ and $g_{*}: N_{\bullet}(\boldsymbol{\Gamma}) \rightarrow N_{\bullet}(\boldsymbol{\Delta})$, for instance see [16, Proposition 4]. In particular equivalent (topological) 2-categories have homotopic nerves. The result follows for a hypercover with a section. Since local sections always exist, the general case reduces to a hypercover $\boldsymbol{\Gamma}\left[\amalg U_{i}\right] \rightarrow \boldsymbol{\Gamma}$ induced by pullback along the canonical map $\coprod U_{i} \rightarrow \boldsymbol{\Gamma}$ where $\left(U_{i}\right)$ is a cover of $\boldsymbol{\Gamma}_{0}$. The result follows from a classical Mayer-Vietoris argument as in [10].

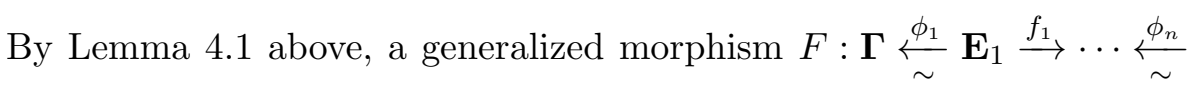
$\mathbf{E}_{n} \stackrel{f_{n}}{\longrightarrow} \boldsymbol{\Delta}$ induces a pullback map in cohomology

$$
F^{*}: H^{\bullet}(\boldsymbol{\Delta}) \stackrel{f_{n}^{*}}{\longrightarrow} H^{\bullet}\left(\boldsymbol{E}_{n}\right) \stackrel{\left(\phi_{n}^{*}\right)^{-1}}{\longrightarrow} \cdots \stackrel{f_{1}^{*}}{\longrightarrow} H^{\bullet}\left(\boldsymbol{E}_{1}\right) \stackrel{\left(\phi_{1}^{*}\right)^{-1}}{\longrightarrow} H^{\bullet}(\boldsymbol{\Gamma})
$$

Clearly, $(F \circ G)^{*}=G^{*} \circ F^{*}$ and, if $F$ is a Morita equivalence, then $F^{*}$ is an isomorphism.

Lemma 4.2. If $F$ and $G$ are equivalent generalized morphisms from $\boldsymbol{\Gamma}$ to $\boldsymbol{\Delta}$, the maps $F^{*}$ and $G^{*}$, which they induce at the cohomology level, are equal. 
Proof. A natural transformation between two 2-functors $f$ and $g$ from $\boldsymbol{\Gamma}$ to $\boldsymbol{\Delta}$ induces a simplicial homotopy between $f_{*}: N_{\bullet}(\boldsymbol{\Gamma}) \rightarrow N_{\bullet}(\boldsymbol{\Delta})$ and $g_{*}$ : $N_{\bullet}(\boldsymbol{\Gamma}) \rightarrow N_{\bullet}(\boldsymbol{\Delta})$ (see [16]). Therefore the lemma follows from the definition of equivalence of generalized morphisms and Lemma 4.1 .

Remark 4.3. Note that, for a Lie 2-groupoid $\Gamma: \Gamma_{2} \frac{l}{u} \gtrless \Gamma_{1} \stackrel{s}{t} \gtrless \Gamma_{0}$, $N_{2} \Gamma$ may be identified to $\Gamma_{2} \times_{s, \Gamma_{0}, t} \Gamma_{1}$ so that the face maps take the form

$$
\begin{gathered}
d_{0}: N_{2} \boldsymbol{\Gamma} \rightarrow N_{1} \boldsymbol{\Gamma}:(\alpha, c) \mapsto u(\alpha) \\
d_{1}: N_{2} \boldsymbol{\Gamma} \rightarrow N_{1} \boldsymbol{\Gamma}:(\alpha, c) \mapsto l(\alpha) \cdot c \\
d_{2}: N_{2} \boldsymbol{\Gamma} \rightarrow N_{1} \boldsymbol{\Gamma}:(\alpha, c) \mapsto c
\end{gathered}
$$

More precisely,

is identified to
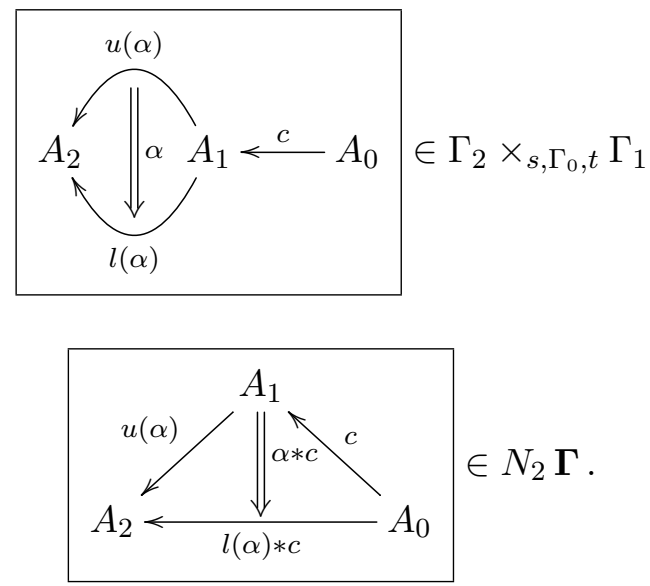

Remark 4.4. The singular cohomology $H_{\text {sing }}^{\bullet}(\boldsymbol{\Gamma}, R)$ of $\boldsymbol{\Gamma}$ with coefficients in a ring $R$ is defined similarly to the de Rham cohomology. More precisely it is the cohomology of $\left(C_{\text {sing }}^{\bullet}\left(N_{\bullet} \Gamma\right), d_{\text {sing }}+\partial\right)$ where $\left(C_{\text {sing }}^{\bullet}(X, R), d_{\text {sing }}\right)$ denotes the singular cochain complex of a space $X$ with coefficients in $R$. As for manifolds, for $R=\mathbb{R}$, one has a natural isomorphism $H_{\text {sing }}(\boldsymbol{\Gamma}, \mathbb{R}) \cong H^{\bullet}(\boldsymbol{\Gamma})$.

\subsection{Cohomology characteristic map for 2-group bundles}

Fix a crossed module $G \rightarrow H$ and let $\mathfrak{B}$ be a principal $[G \rightarrow H]$-bundle over $\boldsymbol{\Gamma}$. In this section we construct a universal characteristic homomorphism $\boldsymbol{C} \boldsymbol{C}_{\mathfrak{B}}: H^{\bullet}([G \rightarrow H]) \rightarrow H^{\bullet}(\boldsymbol{\Gamma})$ generalizing the usual characteristic classes of a principal bundle. 
By definition, $\mathfrak{B}$ is a generalized morphism $\boldsymbol{\Gamma} \rightsquigarrow[G \rightarrow H]$. Therefore, passing to cohomology, we obtain the homomorphism

$$
\boldsymbol{C} \boldsymbol{C}_{\mathfrak{B}}: H^{\bullet}([G \rightarrow H]) \stackrel{\mathfrak{B}^{*}}{\longrightarrow} H^{\bullet}(\boldsymbol{\Gamma})
$$

which we call the characteristic homomorphism of the $[G \rightarrow H]$-bundle $\mathfrak{B}$. It depends only on the isomorphism class of the 2-group bundle.

Proposition 4.5. If $\mathfrak{B}$ and $\mathfrak{B}^{\prime}$ are isomorphic $[G \rightarrow H]$-bundles over $\boldsymbol{\Gamma}$, then $\boldsymbol{C} \boldsymbol{C}_{\mathfrak{B}}=\boldsymbol{C} \boldsymbol{C}_{\mathfrak{B}^{\prime}}: H^{\bullet}([G \rightarrow H]) \rightarrow H^{\bullet}(\boldsymbol{\Gamma})$.

Proof. It is an immediate consequence of Lemma 4.1 since isomorphic principal 2-group bundles are equivalent as generalized morphisms.

Remark 4.6. By analogy with the case of principal bundles, one can think of the elements of $H^{\bullet}([G \rightarrow H])$ as universal characteristic classes and their images in $H^{\bullet}(\boldsymbol{\Gamma})$ by $\boldsymbol{C}_{\mathfrak{B}}$ as characteristic classes of the $[G \rightarrow H]$-bundle over $\boldsymbol{\Gamma}$.

For instance, it is proved [25, Proposition 6.3] that the characteristic classes associated to the string 2-group associated to a compact simple Lie group coincide with the usual ones modulo the Pontryagin class.

Example 4.7. Let $P \stackrel{\pi}{\rightarrow} M$ be a principal $H$-bundle. Then, by Example 2.23, $P$ induces a structure of $[1 \rightarrow H]$-bundle over $M$. Since $H^{\bullet}([1 \rightarrow$ $H]) \cong H^{\bullet}(B H)$, the characteristic map $\boldsymbol{C} \boldsymbol{C}_{P}$ of this bundle coincides with the classical map $H^{\bullet}(B H) \rightarrow H^{\bullet}(M)$ induced by the principal $H$-bundle structure on $P$. In particular, for a compact Lie group $H$, the characteristic map coincides with the Chern-Weil map $S\left(\mathfrak{h}^{*}\right)^{\mathfrak{h}} \rightarrow H^{\bullet}(M)$ induced by the choice of a connection on $P$.

Example 4.8. From Example 2.27, we know that the inertia groupoid of a Lie groupoid $\boldsymbol{\Gamma}$ gives rise to a principal $[\mathbb{Z} \rightarrow \mathbb{R}]$-bundle. In that case $H^{\bullet}([\mathbb{Z} \rightarrow \mathbb{R}]) \cong H^{\bullet}\left(B S^{1}\right) \cong \mathbb{R}[x]$ where $x$ is a generator of degree 2 . In particular, we get a characteristic class $\boldsymbol{C C}_{\Gamma}(x) \in H^{2}\left(\left[\Lambda \boldsymbol{\Gamma} / S^{1}\right]\right)=H_{S^{1}}^{2}(\Lambda \boldsymbol{\Gamma})$ (see [24] for equivariant cohomology of stacks). For instance if $\boldsymbol{\Gamma}$ is the groupoid $G \rightrightarrows 1$ with $G$ a simply connected compact Lie group, then its inertia groupoid is the transformation Lie groupoid $G \times G \rightrightarrows G$ with $G$ acting on itself by the adjoint action. From the Gysin sequence in equivariant homology of stacks [24, $\S 8]$ and the fact that the homology $H^{\bullet}(G \times G \rightrightarrows G)$ 
is trivial in degrees 1 and 2 , we see that $\boldsymbol{C} \boldsymbol{C}_{G \times G \rightrightarrows G}(x)$ is an integra $\bigsqcup^{6}$ generator of $H^{2}\left(\left[\Lambda \boldsymbol{\Gamma} / S^{1}\right]\right)$.

Let $\boldsymbol{\Gamma} \stackrel{F}{\rightsquigarrow} \boldsymbol{\Delta}$ be a generalized morphism of Lie (1-)groupoids and let $\mathfrak{B}$ : $\boldsymbol{\Delta} \underset{\sim}{\stackrel{\phi}{\sim}} \boldsymbol{E} \stackrel{f}{\rightarrow}[G \rightarrow H]$ be a 2 -group bundle with base $\boldsymbol{\Delta}$. The pullback $F^{*}(\mathfrak{B})$ of the $[G \rightarrow H]$-bundle $\mathfrak{B}$ from $\boldsymbol{\Delta}$ to $\boldsymbol{\Gamma}$ by $F$ is the composition $\mathfrak{B} \circ F$ of the two generalized morphisms. It is a principal $[G \rightarrow H]$-bundle over $\boldsymbol{\Gamma}$.

The Whitney sum of two 2-group bundles is defined as follows. Let $\mathfrak{B}$ : $\boldsymbol{\Gamma} \stackrel{\phi}{\stackrel{\sim}{\sim}} \boldsymbol{E} \stackrel{f}{\rightarrow}[G \rightarrow H]$ and $\mathfrak{B}^{\prime}: \boldsymbol{\Gamma} \underset{\sim}{\stackrel{\phi^{\prime}}{\leftarrow}} \boldsymbol{E}^{\prime} \stackrel{f^{\prime}}{\rightarrow}\left[G^{\prime} \rightarrow H^{\prime}\right]$ be two 2-group bundles over the same base $\boldsymbol{\Gamma}$. Let $\boldsymbol{F}$ be the "fiber product" 2-groupoid $E_{2} \times_{\Gamma_{2}}$ $E_{2}^{\prime} \rightrightarrows E_{1} \times_{\Gamma_{1}} E_{1}^{\prime} \rightrightarrows E_{0} \times_{\Gamma_{0}} E_{0}^{\prime}$ with the obvious structure maps: $s\left(e, e^{\prime}\right)=$ $\left(s(e), s\left(e^{\prime}\right)\right),\left(x, x^{\prime}\right) *\left(y, y^{\prime}\right)=\left(x * x^{\prime}, y * y^{\prime}\right)$, etc. The Whitney sum $\mathfrak{B} \oplus \mathfrak{B}^{\prime}$ is the $\left[G \times G^{\prime} \rightarrow H \times H^{\prime}\right]$-bundle over $\boldsymbol{\Gamma}$ given by the generalized morphism $\boldsymbol{\Gamma} \underset{\sim}{\stackrel{\phi=\phi^{\prime}}{\sim}} \boldsymbol{F} \stackrel{f \times f^{\prime}}{\longrightarrow}\left[G \times G^{\prime} \rightarrow H \times H^{\prime}\right]$

By Proposition 4.5, we obtain

Corollary 4.9. 1) $C C_{F^{*}(\mathfrak{B})}=F^{*} \circ C C_{\mathfrak{B}}$.

2) $\boldsymbol{C}_{\mathfrak{B} \oplus \mathfrak{B}^{\prime}}=\Delta^{*} \circ\left(\boldsymbol{C} \boldsymbol{C}_{\mathfrak{B}} \times \boldsymbol{C}_{\mathfrak{B}^{\prime}}\right)$, where $\Delta: \boldsymbol{\Gamma} \rightarrow \boldsymbol{\Gamma} \times \boldsymbol{\Gamma}$ is the diagonal map and $\times$ is the cross-product $H^{\bullet}([G \rightarrow H]) \otimes H^{\bullet}\left(\left[G^{\prime} \rightarrow H^{\prime}\right]\right) \cong$ $H^{\bullet}\left(\left[G \times G^{\prime} \rightarrow H \times H^{\prime}\right]\right)$.

Remark 4.10. The result of this section easily extends to singular cohomology with any coefficient (see Remark 4.4). In particular the characteristic map

$$
\boldsymbol{C} \boldsymbol{C}_{\mathfrak{B}}: H_{\text {sing }}^{\bullet}([G \rightarrow H], \mathbb{Z}) \stackrel{\mathfrak{B}^{*}}{\longrightarrow} H^{\bullet}(\boldsymbol{\Gamma}, \mathbb{Z})
$$

is defined in cohomology with integer coefficients.

Remark 4.11. By Proposition 3.8 , a Lie groupoid $G$-extension $\tilde{\Gamma} \stackrel{\phi}{\rightarrow} \Gamma \rightrightarrows$ $M$ induces a principal $[G \rightarrow \operatorname{Aut}(G)]$-bundle $\mathfrak{B}_{\phi}$ over the groupoid $\Gamma \rightrightarrows$ $M$. Hence we obtain the universal characteristic map $\boldsymbol{C C}_{\mathfrak{B}_{\phi}}: H^{\bullet}([G \stackrel{\text { Ad }}{\longrightarrow}$ $\operatorname{Aut}(G)]) \rightarrow H^{\bullet}(\boldsymbol{\Gamma})$. Unfortunately, the cohomology $H^{\bullet}([G \stackrel{\text { Ad }}{\longrightarrow} \operatorname{Aut}(G)])$ is not known when the center of $G$ is large and it is trivial when the center of $G$ is of dimension less than three [25]. Therefore one cannot have much hope of getting interesting characteristic classes except for extensions whose structure 2-group can be reduced. Indeed, this is the object of the next section.

\footnotetext{
${ }^{6}$ More precisely, it is the image of a generator of the cohomology with integer coefficients. See Remark 4.10 .
} 


\subsection{DD classes for groupoid central $G$-extensions}

Let $\tilde{\Gamma} \stackrel{\phi}{\rightarrow} \Gamma \rightrightarrows M$ be a $G$-extension of Lie groupoids. Let $\phi^{\prime}$ denote the factorization of the morphism $\phi$ through the projection $q: \tilde{\Gamma} \rightarrow \tilde{\Gamma} / Z(G)$ :

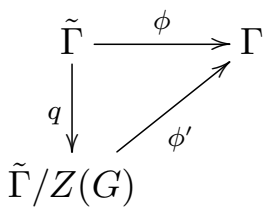

The extension $\phi$ is said to be central 33] if there exists a section $\sigma: \Gamma \rightarrow$ $\tilde{\Gamma} / Z(G)$ of $\phi^{\prime}$ such that

$$
x g=g x \quad \forall x \in q^{-1}(\sigma(\Gamma)), \forall g \in G
$$

In this case, the subspace $\tilde{\Gamma}^{\prime}=q^{-1}(\sigma(\Gamma))$ of $\tilde{\Gamma}$ is a central $Z(G)$-extension of $\Gamma \rightrightarrows M$.

Remark 4.12. The definition of a central $G$-gerbe here is taken from [33]. According to loc. cit, it agrees with the one of a $G$-gerbe with trivial band.

Given $\gamma \in \tilde{\Gamma}$, there exists $x \in \tilde{\Gamma}^{\prime}$ such that $\phi(x)=\phi(\gamma)$. Thus there exists $k \in G$ such that $\gamma=x \cdot k$. Given $\gamma$, both $x$ and $k$ are uniquely determined up to an element of $Z(G)$. Defining a homomorphism of Lie groupoids $r$ : $\tilde{\Gamma} \rightarrow G / Z(G)$ by the relation $q(\gamma)=\sigma(\phi(\gamma)) r(\gamma)$, we obtain that, for any $g \in G$,

$$
g \gamma=g x k=x g k=x k \cdot k^{-1} g k=\gamma g^{r(\gamma)}
$$

where $g^{r(\gamma)}$ denotes the conjugate $k^{-1} g k$ of $g$ by any element $k \in G$ such that $k Z(G)=r(\gamma)$.

Proposition 4.13. Let $\tilde{\Gamma} \stackrel{\phi}{\rightarrow} \Gamma \rightrightarrows M$ be a G-extension of a Lie groupoid $\Gamma$ and let $\mathfrak{B}$ denote the corresponding $[G \rightarrow \operatorname{Aut}(G)]$-bundle. The extension is central if, and only if, the $[G \rightarrow \operatorname{Aut}(G)]$-bundle $\mathfrak{B}$ reduces to a principal $[Z(G) \rightarrow 1]$-bundle, i.e. there exists a generalized morphism $Z \mathfrak{B}:[M \rightarrow$ $\Gamma] \rightarrow[Z(G) \rightarrow 1]$ such that

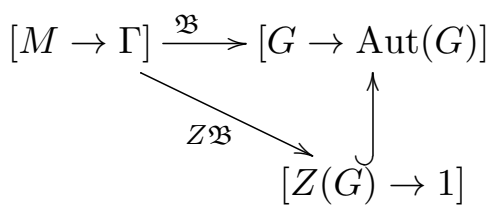

is commutative up to equivalence. 
In particular, being central is invariant under Morita equivalences of Lie groupoids extension.

Proof. Let $\tilde{\Gamma} \stackrel{\phi}{\rightarrow} \Gamma \rightrightarrows M$ be a central $G$-extension. The corresponding 2group bundle $\mathfrak{B}$ is the generalized morphism $[M \rightarrow \Gamma] \leftarrow[M \times G \stackrel{i}{\rightarrow} \tilde{\Gamma}] \rightarrow$ $[G \stackrel{\text { Ad }}{\longrightarrow} \operatorname{Aut}(G)]$, see Proposition 3.8 and Remark 3.7. Let $\tau: \tilde{\Gamma}^{\prime} \rightarrow \tilde{\Gamma}$ be the inclusion map. The $Z(G)$-extension defines the crossed module $[M \times$ $Z(G) \stackrel{i^{\prime}}{\rightarrow} \tilde{\Gamma}^{\prime}$ and we have a commutative diagram

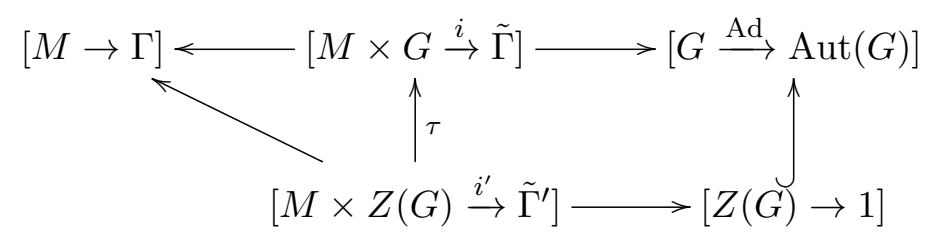

Note that the right square in (15) is commutative because the extension is central. Diagram (15) implies that the 2-group bundle $\mathfrak{B}$ reduces.

Reciprocally, assume $\mathfrak{B}$ reduces. By Proposition 3.102, passing to a Morita equivalent groupoid, we can assume that the $G$-extension is the extension corresponding to the generalized morphism $[M \rightarrow \Gamma] \stackrel{Z \mathfrak{B}}{\rightsquigarrow}[Z(G) \rightarrow$ 1] $\hookrightarrow[G \rightarrow \operatorname{Aut}(G)]$. If $Z \mathfrak{B}$ is the generalized morphism $[M \rightarrow \Gamma] \leftarrow[M \times$ $L \rightarrow \Delta] \rightarrow[Z(G) \rightarrow 1]$, the associated extension is, according to Section 3.2 , $\tilde{\Gamma} \rightarrow \Gamma \rightrightarrows M$, where $\tilde{\Gamma}=\left(\Delta \times{ }_{L} Z(G)\right) \times{ }_{Z(G)} G$. Since the composition $\Delta \rightarrow$ $1 \rightarrow \operatorname{Aut}(G)$ is trivial, $\operatorname{Ad}_{\widetilde{\gamma}}$ is trivial for all $\widetilde{\gamma} \in \tilde{\Gamma}$. Therefore, the extension is central.

Remark 4.14. If $G$ is a Lie group whose Lie algebra $\mathfrak{g}$ is reductive, its Lie algebra decomposes as a direct sum $\mathfrak{g} \cong Z(\mathfrak{g}) \oplus \mathfrak{m}$ of ideals, where $Z(\mathfrak{g})$ is the center of $\mathfrak{g}$. In the sequel, the symbol pr will denote the induced projection $\mathfrak{g} \rightarrow Z(\mathfrak{g})$, which is a homomorphism of Lie algebras and maps $[\mathfrak{g}, \mathfrak{g}]$ onto 0 . Moreover, if $G$ is connected, this direct sum decomposition is not only $\operatorname{ad}_{Z(\mathfrak{g})}$-invariant but also $\operatorname{Ad}_{G}$-invariant and, consequently, pr $\circ \operatorname{Ad}_{g}=$ pr for all $g \in G$. Moreover, for any $g \in G$ and any smooth path $t \mapsto f_{t}$ in $G$ with $f_{0}=1$ and $\left.\frac{d}{d t} f_{t}\right|_{0}=\xi \in \mathfrak{g}$, one has

$$
\operatorname{pr}\left(\left.\frac{d}{d t} f_{t}^{-1} g f_{t} g^{-1}\right|_{0}\right)=\operatorname{pr}\left(\operatorname{Ad}_{g} \xi-\xi\right)=\operatorname{pr}(\xi)-\operatorname{pr}(\xi)=0
$$


Proposition 4.15. Let $\tilde{\Gamma} \stackrel{\phi}{\rightarrow} \Gamma \rightrightarrows M$ be a central $G$-extension with $G$ connected and whose Lie algebra is reductive $\square^{7}$ Let $\alpha \in \Omega^{1}(\tilde{\Gamma} ; \mathfrak{g})$ be a connection 1-form for the right principal G-bundle $\tilde{\Gamma} \stackrel{\phi}{\rightarrow} \Gamma$.

1) Then there exists $\Omega_{\alpha} \in Z_{\mathrm{DR}}^{3}\left(\Gamma_{.} ; Z(\mathfrak{g})\right)$ such that $\operatorname{pr}(d \alpha+\partial \alpha)=\phi^{*}\left(\Omega_{\alpha}\right)$.

2) Moreover, if $\alpha_{1}$ and $\alpha_{2}$ are two different connection 1-forms, then $\Omega_{\alpha_{1}}-\Omega_{\alpha_{2}} \in B_{\mathrm{DR}}^{3}\left(\Gamma_{\bullet} ; Z(\mathfrak{g})\right)$.

We call $\boldsymbol{D} \boldsymbol{D}_{(\alpha)}:=\left[\Omega_{\alpha}\right] \in H^{3}(\Gamma) \otimes Z(\mathfrak{g})$ the Dixmier-Douady class of the G-central extension.

Proof. 1) Being a connection 1-form, $\alpha \in \Omega^{1}(\tilde{\Gamma} ; \mathfrak{g})$ enjoys the following two properties:

$$
\begin{gathered}
R_{g}^{*} \alpha=\operatorname{Ad}_{g^{-1}} \circ \alpha, \quad \forall g \in G \\
\alpha\left(\hat{\xi}_{x}\right)=\xi, \quad \forall x \in M, \forall \xi \in \mathfrak{g}
\end{gathered}
$$

Given any $\xi \in \mathfrak{g}$ and any $G$-invariant vector field $v \in \mathfrak{X}(\tilde{\Gamma})$, we get

$$
\begin{aligned}
d \alpha(\hat{\xi}, v)=\hat{\xi}(\alpha(v))-v(\alpha(\hat{\xi}))-\alpha([\hat{\xi}, v]) & =\hat{\xi}(\alpha(v))-v(\xi)-\alpha\left(\mathcal{L}_{\hat{\xi}} v\right) \\
& =\mathcal{L}_{\hat{\xi}}(\alpha(v))=-\operatorname{ad}_{\xi}(\alpha(v))
\end{aligned}
$$

since the vector field $v$ is $G$-invariant and the function $\alpha(v)$ is $G$-equivariant. It follows that $\operatorname{pr} \circ d \alpha(\hat{\xi}, v)=\operatorname{pr}[\alpha(v), \xi]=0$ since $\operatorname{pr}[\mathfrak{g}, \mathfrak{g}]=0$. Moreover, we have

$$
R_{g}^{*}(d \alpha)=d\left(R_{g}^{*} \alpha\right)=d\left(\operatorname{Ad}_{g^{-1}} \circ \alpha\right)=\operatorname{Ad}_{g^{-1}} \circ d \alpha
$$

for all $g \in G$. Therefore, by Remark 4.14 , the 2 -form $\operatorname{pr} \circ d \alpha \in \Omega^{2}(\tilde{\Gamma}, Z(\mathfrak{g}))$ is basic; there exists $\omega \in \Omega^{2}(\Gamma, Z(\mathfrak{g}))$ such that pr $\circ d \alpha=\phi^{*} \omega$.

Consider

$$
\tilde{\Gamma}_{2}=\tilde{\Gamma} \times_{s, \Gamma, t} \tilde{\Gamma}=\{(x, y) \in \tilde{\Gamma} \times \tilde{\Gamma} \mid s(x)=t(y)\},
$$

the three face maps

$$
p_{1}(x, y)=x \quad m(x, y)=x \cdot y \quad p_{2}(x, y)=y
$$

${ }^{7}$ Such Lie groups are called reductive, though this terminology sometimes applies only to algebraic groups. Examples of Lie groups with reductive Lie algebras include $G L_{n}(\mathbb{R})$ and all compact Lie groups. 
from $\tilde{\Gamma}_{2}$ to $\tilde{\Gamma}$ and the action of $G \times G$ on $\tilde{\Gamma}_{2}$ given by

$$
(x, y)^{(g, h)}=\left(x g_{s(x)}, y h_{s(y)}\right)
$$

Then we have

$$
\operatorname{pr} \circ \partial \alpha=\partial(\operatorname{pr} \circ \alpha)=p_{2}^{*}(\operatorname{pr} \circ \alpha)-m^{*}(\operatorname{pr} \circ \alpha)+p_{1}^{*}(\operatorname{pr} \circ \alpha)
$$

From $\operatorname{pr} \circ \operatorname{Ad}_{g}=\operatorname{pr}$ and $R_{g}^{*} \alpha=\operatorname{Ad}_{g^{-1}} \alpha$, it follows that $R_{g}^{*}(\operatorname{pr} \circ \alpha)=\operatorname{pr} \circ \alpha$. This, together with the relations $p_{2} \circ R_{(g, h)}=R_{h} \circ p_{2}$ and $p_{1} \circ R_{(g, h)}=R_{g} \circ$ $p_{1}$ implies that $p_{2}^{*}(\operatorname{pr} \circ \alpha)$ and $p_{1}^{*}(\operatorname{pr} \circ \alpha)$ are invariant under the $G \times G$-action $\tilde{\Gamma}_{2}$. Given a smooth path $t \mapsto\left(x_{t}, y_{t}\right)$ in $\tilde{\Gamma}_{2}$, one also gets

$$
\begin{aligned}
& R_{(g, h)}^{*} m^{*}(\operatorname{pr} \circ \alpha)\left(\left.\frac{d}{d t}\left(x_{t}, y_{t}\right)\right|_{0}\right) \\
= & (\operatorname{pr} \circ \alpha)\left(\left.\frac{d}{d t} x_{t} g y_{t} h\right|_{0}\right) \\
= & (\operatorname{pr} \circ \alpha)\left(\left.\frac{d}{d t} x_{t} y_{t} g^{r\left(y_{t}\right)} h\right|_{0}\right) \\
= & (\operatorname{pr} \circ \alpha)\left(\left.\frac{d}{d t} x_{t} y_{t} g^{r\left(y_{0}\right)} h\right|_{0}\right)+(\operatorname{pr} \circ \alpha)\left(\left.\frac{d}{d t} x_{0} y_{0} g^{r\left(y_{t}\right)} h\right|_{0}\right)
\end{aligned}
$$

While the first term of the r.h.s. is equal to $m^{*}(\operatorname{pr} \circ \alpha)\left(\left.\frac{d}{d t}\left(x_{t}, y_{t}\right)\right|_{0}\right)$ since ( $\mathrm{pr} \circ \alpha$ ) is $G$-invariant, the second term vanishes. Indeed, using $\alpha(\hat{\xi})=\xi$, $R_{h}^{*} \alpha=\operatorname{Ad}_{h^{-1}} \circ \alpha$ and $\mathrm{pr} \circ \operatorname{Ad}_{g}=$ pr, we obtain that

$$
\begin{aligned}
(\operatorname{pr} \circ \alpha)\left(\left.\frac{d}{d t} x_{0} y_{0} g^{r\left(y_{t}\right)} h\right|_{0}\right) & =\operatorname{pr}\left(\left.\frac{d}{d t} g^{r\left(y_{t}\right)}\left(g^{r\left(y_{0}\right)}\right)^{-1}\right|_{0}\right) \\
& =\operatorname{pr}\left(\left.\frac{d}{d t}\left(g^{r\left(y_{0}\right)}\right)^{r\left(y_{0}\right)^{-1} r\left(y_{t}\right)}\left(g^{r\left(y_{0}\right)}\right)^{-1}\right|_{0}\right)
\end{aligned}
$$

and the claim follows from 16 . Hence $R_{(g, h)}^{*} m^{*}(\operatorname{pr} \circ \alpha)=m^{*}(\operatorname{pr} \circ \alpha)$. Therefore, $\operatorname{pr} \circ \partial \alpha$ is $(G \times G)$-invariant.

One also has

$$
\begin{aligned}
\operatorname{pr} \circ \partial \alpha\left(\left.\frac{d}{d t}\left(x e^{t \xi}, y e^{t \eta}\right)\right|_{0}\right) & =\operatorname{pr}\left(\alpha\left(\left.\frac{d}{d t} y e^{t \eta}\right|_{0}\right)-\alpha\left(\left.\frac{d}{d t} x e^{t \xi} y e^{t \eta}\right|_{0}\right)+\alpha\left(\left.\frac{d}{d t} x e^{t \xi}\right|_{0}\right)\right) \\
& =\operatorname{pr}\left(\eta-\alpha\left(\left.\frac{d}{d t} x y e^{t \operatorname{Ad}_{r(y)}^{-1} \xi} e^{t \eta}\right|_{0}\right)+\xi\right) \\
& =\operatorname{pr}\left(\eta-\operatorname{Ad}_{r(y)}^{-1} \xi-\eta+\xi\right) \\
& =\operatorname{pr}(\xi)-\operatorname{pr}\left(\operatorname{Ad}_{r(y)}^{-1} \xi\right) \\
& =0 .
\end{aligned}
$$


Hence the 1-form pr $\circ \partial \alpha \in \Omega^{1}\left(\tilde{\Gamma}_{2}, Z(\mathfrak{g})\right)$ is basic with respect to the principal $(G \times G)$-bundle $\tilde{\Gamma}_{2} \rightarrow \Gamma_{2}$.

2 Clearly, one has $i_{\hat{\xi}}\left(\alpha_{1}-\alpha_{2}\right)=0$ and $R_{g}^{*}\left(\operatorname{pr} \circ\left(\alpha_{1}-\alpha_{2}\right)\right)=\operatorname{pr} \circ\left(\alpha_{1}-\right.$ $\left.\alpha_{2}\right)$. Thus pro( $\left.\alpha_{1}-\alpha_{2}\right) \stackrel{\xi}{=} \phi^{*} A$, where $A \in \Omega^{1}(\Gamma ; Z(\mathfrak{g}))$. It follows that

$$
\begin{aligned}
\phi^{*}\left(\Omega_{\alpha_{1}}-\Omega_{\alpha_{2}}\right) & =\operatorname{pr}\left(d\left(\alpha_{1}-\alpha_{2}\right)+\partial\left(\alpha_{1}-\alpha_{2}\right)\right) \\
& =d\left(\phi^{*} A\right)+\partial\left(\phi^{*} A\right)=\phi^{*}(d A+\partial A)
\end{aligned}
$$

and $\Omega_{\alpha_{1}}-\Omega_{\alpha_{2}}=d A+\partial A \in B^{3}\left(\Gamma_{\bullet} ; Z(\mathfrak{g})\right)$.

Remark 4.16. The Dixmier-Douady class $\boldsymbol{D} \boldsymbol{D}_{(\alpha)}$ of a central $G$-extension identifies with a linear map $Z(\mathfrak{g})^{*} \rightarrow H^{3}(\boldsymbol{\Gamma})$ by composition with the canonical biduality homomorphism $Z(\mathfrak{g}) \rightarrow Z(\mathfrak{g})^{* *}$.

Remark 4.17. When the group $G$ is abelian, then $\tilde{\Gamma} / Z(G)=\Gamma, \tilde{\Gamma}^{\prime}=\tilde{\Gamma}$ and the projection map $\mathfrak{g} \stackrel{\text { pr }}{\rightarrow} Z(\mathfrak{g})=\mathfrak{g}$ is the identity. In particular, when $G=S^{1}$, the Dixmier-Douady class given by Proposition 4.15 coincides with the Dixmier-Douady class defined in [8].

\subsection{Main theorem}

Let $\tilde{\Gamma} \rightarrow \Gamma \rightrightarrows M$ be a central $G$-extension of Lie groupoids.

According to Proposition 4.13, we obtain a universal characteristic map $\boldsymbol{C C}_{\Phi}: H^{3}([Z(G) \rightarrow 1]) \rightarrow H^{3}(\boldsymbol{\Gamma})$. According to $\left[25, H^{3}([Z(G) \rightarrow 1])\right.$ is isomorphic to $Z(\mathfrak{g})^{*}$ if $G$ is compact. Thus we obtain a map $\boldsymbol{C C}_{\Phi}: Z(\mathfrak{g})^{*} \rightarrow$ $H^{3}(\boldsymbol{\Gamma})$ which, by duality, defines the universal characteristic class $\boldsymbol{C C}_{\Phi} \in$ $H^{3}(\boldsymbol{\Gamma}) \otimes Z(\mathfrak{g})$.

Our main theorem is

Theorem 4.18. Let $G$ be a compact connected Lie group. For any central $G$-extension of Lie groupoids $\tilde{\Gamma} \rightarrow \Gamma \rightrightarrows M$, the universal characteristic class coincides with the Dixmier-Douady class.

Remark 4.19. The theorem above may be considered as a higher analogue of Chern-Weil theory where the characteristic classes of a principal bundle can be expressed by geometric data such as connection and curvature. In particular when $G$ is $S^{1}$, Theorem 4.18 is a higher analogue of the following well known fact: the Chern class of an $S^{1}$-bundle can be computed from its curvature. The latter played an important role in geometric quantization of 
symplectic manifolds. We refer the interested reader to [57] for prequantization of symplectic groupoids, which may be interpreted as a construction of a central $S^{1}$-extension whose Dixmier-Douady class is the prescribed symplectic form.

As a corollary we obtain

Corollary 4.20. Let $G$ be a compact connected Lie group. The DixmierDouady class of any central G-extension of Lie groupoids is an integral class.

Proof. The singular cohomology with integer coefficients $H^{\bullet}(\boldsymbol{\Gamma} ; \mathbb{Z})$ of a 2 groupoid $\boldsymbol{\Gamma}$ is defined as for de Rham cohomology, substituting the singular cochain complex to the de Rham forms in the constructions of Section 4.1, see Remark 4.4. Therefore, given any principal $[Z(G) \rightarrow 1]$-bundle $\mathfrak{B}$ over $\boldsymbol{\Gamma}$, we can construct an integer valued universal characteristic homomorphism $\boldsymbol{C C}_{\mathfrak{B}}: H^{\bullet}([Z(G) \rightarrow 1], \mathbb{Z}) \rightarrow H^{\bullet}(\boldsymbol{\Gamma}, \mathbb{Z})$ as in Section 4.2, see Remark 4.10. According to the computations in [25], the image of $H^{3}([Z(G) \rightarrow 1], \mathbb{Z})$ under the canonical morphism $H^{3}([Z(G) \rightarrow 1], \mathbb{Z}) \rightarrow H^{3}([Z(G) \rightarrow 1])$ is the lattice in $Z(\mathfrak{g})^{*}$ generated by the fundamental classes of each circle component of $Z(G) \cong S^{1} \times \cdots \times S^{1}$. Now the result follows from Theorem 4.18 .

\subsection{The case of central $S^{1}$-extensions}

In this section, we establish Theorem 4.18 in the case $G=S^{1}$.

Assume $\tilde{\Gamma} \stackrel{\phi}{\rightarrow} \Gamma \rightrightarrows M$ is a central $S^{1}$-extension. We consider the following four 2-groupoids:

$$
\begin{aligned}
& \boldsymbol{A}: \quad \Gamma \rightrightarrows \Gamma \rightrightarrows M \quad \boldsymbol{B}: \quad \tilde{\Gamma} \times_{\Gamma} \tilde{\Gamma} \rightrightarrows \tilde{\Gamma} \rightrightarrows M \\
& \boldsymbol{C}: \quad Z(G) \rightrightarrows * \rightrightarrows * \quad \boldsymbol{D}: \quad \tilde{\Gamma} \rightrightarrows \tilde{\Gamma} \rightrightarrows M
\end{aligned}
$$

The central extension $\phi$ determines the (generalized) morphisms

$$
\boldsymbol{D} \stackrel{\phi}{\longrightarrow} \boldsymbol{A} \quad \text { and } \quad \boldsymbol{A} \stackrel{\phi}{\sim} \boldsymbol{B} \stackrel{f}{\longrightarrow} \boldsymbol{C}
$$

At the nerve level, we get

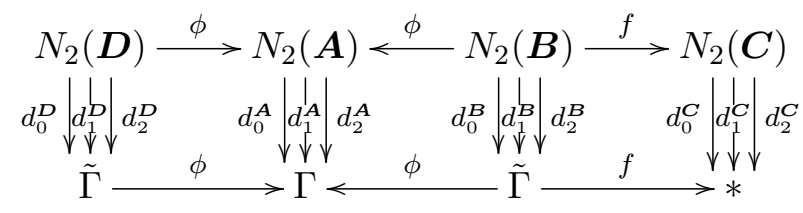


where, according to Remark 4.3 .

$$
\begin{gathered}
N_{2}(\boldsymbol{A})=\Gamma \times_{t, \Gamma_{0}, s} \Gamma, \quad N_{2}(\boldsymbol{C})=Z(G), \quad N_{2}(\boldsymbol{D})=\tilde{\Gamma} \times_{t, \Gamma_{0}, s} \tilde{\Gamma}, \\
N_{2}(\boldsymbol{B})=\left\{(a, b, c) \in \tilde{\Gamma}^{3} \mid \phi(a)=\phi(b) \text { and } s(a)=s(b)=t(c)\right\} \\
\phi: N_{2}(\boldsymbol{B}) \rightarrow N_{2}(\boldsymbol{A}):(a, b, c) \mapsto(\phi(a), \phi(c)) \\
f: N_{2}(\boldsymbol{B}) \rightarrow N_{2}(\boldsymbol{C}):(a, b, c) \mapsto a b^{-1}
\end{gathered}
$$

and the face maps are given by

$$
\begin{aligned}
& d_{0}^{\boldsymbol{A}}(a, c)=a \\
& d_{1}^{\boldsymbol{A}}(a, c)=a c \\
& d_{2}^{A}(a, c)=c \\
& d_{0}^{\boldsymbol{B}}(a, b, c)=a \\
& d_{1}^{\boldsymbol{B}}(a, b, c)=b c \\
& d_{2}^{\boldsymbol{B}}(a, b, c)=c \\
& d_{0}^{\boldsymbol{D}}(a, c)=a \\
& d_{1}^{\boldsymbol{D}}(a, c)=a c \\
& d_{2}^{B}(a, c)=c
\end{aligned}
$$

We will need one more map:

$$
p_{13}: N_{2}(\boldsymbol{B}) \rightarrow N_{2}(\boldsymbol{D}):(a, b, c) \mapsto(a, c)
$$

Lemma 4.21. One has

(18) and $\quad d_{1}^{\boldsymbol{D}} \circ p_{13}(a, b, c)=f(a, b, c) \cdot d_{1}^{\boldsymbol{B}}(a, b, c), \quad \forall(a, b, c) \in N_{2}(\boldsymbol{B})$.

Lemma 4.22. For any pseudo-connection $\theta \in \Omega(\tilde{\Gamma})$ on the central $S^{1}$ extension $\tilde{\Gamma} \stackrel{\phi}{\rightarrow} \Gamma \rightrightarrows \Gamma_{0}$, one has

$$
\partial^{\boldsymbol{B}} \theta+f^{*}(d t)=p_{13}^{*}\left(\partial^{\boldsymbol{D}} \theta\right)
$$

Here dt denotes the Maurer-Cartan (or angular) form on $S^{1}$.

Proof. Since $\theta\left(\left.\frac{d}{d t} \tilde{\gamma} \cdot e^{i t}\right|_{0}\right)=1$ and $\theta$ is $S^{1}$-invariant, it follows from 18 that

$$
\left(d_{1}^{\boldsymbol{D}} \circ p_{13}\right)^{*} \theta=\left(d_{1}^{\boldsymbol{B}}\right)^{*} \theta+f^{*} d t
$$

Therefore,

$$
\begin{aligned}
\partial^{\boldsymbol{B}} \theta-p_{13}^{*}\left(\partial^{\boldsymbol{D}} \theta\right) & =\left(d_{1}^{\boldsymbol{B}}\right)^{*} \theta-p_{13}^{*}\left(d_{1}^{\boldsymbol{D}}\right)^{*} \theta & & \text { by }(17), \\
& =-f^{*} d t & & \text { by }(19) .
\end{aligned}
$$

According to Proposition 4.15, the connection $\theta$ induces a cocycle $\Omega_{\theta} \in$ $Z_{\mathrm{DR}}^{3}(\boldsymbol{A})$. 
Theorem 4.23. $\phi^{*}\left[\Omega_{\theta}\right]=f^{*}[d t]$ in $H^{3}(\boldsymbol{B})$

Proof. By construction, the cocycle $\Omega_{\theta}$ is the sum $\Omega_{\theta}=\eta+\omega$, where $\phi^{*}(\eta)=$ $\partial^{\boldsymbol{D}} \theta$ and $\phi^{*}(\omega)=d_{\mathrm{DR}} \theta$.

$$
\begin{aligned}
\partial^{\boldsymbol{B}} \theta+d \theta & =p_{13}^{*}\left(\partial^{\boldsymbol{D}} \theta\right)-f^{*}(d t)+d \theta \quad \text { by Lemma } 4.22 \\
& =p_{13}^{*}\left(\phi^{*} \eta\right)-f^{*}(d t)+\phi^{*} \omega \\
& =\phi^{*}(\eta+\omega)-f^{*}(d t)
\end{aligned}
$$

Now, Theorem 4.18 in the case $G=S^{1}$ follows from Theorem 4.23 since $\boldsymbol{C} \boldsymbol{C}_{\phi}=\left(\phi^{*}\right)^{-1}\left(f^{*}(d t)\right)$ and $\boldsymbol{D} \boldsymbol{D}_{(\phi)}=\left[\Omega_{\theta}\right]$ (by Proposition 4.15).

\subsection{Proof of Theorem 4.18}

By [33] (see also Section 4.3), the central $G$-extension of Lie groupoids $\tilde{\Gamma} \rightarrow \Gamma \rightrightarrows M$ induces a central $Z(G)$-extension $\tilde{\Gamma}^{\prime} \rightarrow \Gamma \rightrightarrows M$, where $\tilde{\Gamma}^{\prime}=$ $q^{-1}(\sigma(\Gamma))$. We recover $\tilde{\Gamma}$ from $\tilde{\Gamma}^{\prime}$ by the formula $\tilde{\Gamma} \cong \frac{\tilde{\Gamma}^{\prime} \times G}{Z(G)}$, where the $Z(G)$ action on $\tilde{\Gamma}^{\prime} \times G$ is given by $(x, g) \cdot z=\left(x \cdot z^{-1}, z \cdot g\right)$ for $x \in \tilde{\Gamma}^{\prime}, g \in G$ and $z \in Z(G)$. The natural inclusion $\tau: \tilde{\Gamma}^{\prime} \rightarrow \tilde{\Gamma}$ coincides with the map $x \mapsto$ $\left[x, 1_{G}\right] \in \frac{\tilde{\Gamma}^{\prime} \times G}{Z(G)}$. Since $G$ is compact, $Z(\mathfrak{g})$ is reductive and we have the Lie algebra morphism pr $: \mathfrak{g} \rightarrow Z(\mathfrak{g})$.

Lemma 4.24. Let $\alpha \in \Omega^{1}(\tilde{\Gamma}, \mathfrak{g})$ be a connection 1-form on the right principal $G$-bundle $\tilde{\Gamma} \rightarrow \Gamma$. Then $\alpha^{\prime}:=\operatorname{pr}\left(\tau^{*}(\alpha)\right) \in \Omega^{1}\left(\tilde{\Gamma}^{\prime}, Z(\mathfrak{g})\right)$ is a connection 1 -form for the right principal $Z(G)$-bundle $\tilde{\Gamma}^{\prime} \rightarrow \Gamma$.

Proof. Since the inclusion $\tau: \tilde{\Gamma}^{\prime} \hookrightarrow \tilde{\Gamma}$ is $Z(G)$-equivariant, we have

$$
\alpha^{\prime}\left(\hat{\eta}_{x}\right)=\operatorname{pr} \circ \alpha \circ \tau_{*}\left(\hat{\eta}_{x}\right)=\operatorname{pr} \circ \alpha \circ\left(\hat{\eta}_{\tau(x)}\right)=\operatorname{pr}(\eta)=\eta
$$

for all $\eta \in Z(\mathfrak{g})$ and $x \in \tilde{\Gamma}^{\prime}$. Similarly, for any $h \in Z(G)$, we have

$$
R_{h}^{*}\left(\alpha^{\prime}\right)=R_{h}^{*}\left(\operatorname{pr}\left(\tau^{*}(\alpha)\right)\right)=\operatorname{pr} \tau^{*} R_{h}^{*} \alpha=\operatorname{pr} \tau^{*} A d_{h^{-1}} \alpha=\alpha^{\prime} .
$$

Since $\tilde{\Gamma}^{\prime} \rightarrow \Gamma \rightrightarrows M$ is a $Z(G)$-central extension, by Lemma 4.24 and Proposition 4.15, we have the Dixmier-Douady class $\boldsymbol{D} \boldsymbol{D}_{\left(\alpha^{\prime}\right)} \in H^{3}(\Gamma) \otimes$ $Z(\mathfrak{g})$.

Proposition 4.25. We have $\boldsymbol{D} \boldsymbol{D}_{\left(\alpha^{\prime}\right)}=\boldsymbol{D} \boldsymbol{D}_{(\alpha)}$. 
Proof. By Lemma 4.24 and Proposition 4.15.(b), we can use the 1-form $\alpha^{\prime}$ to calculate the Dixmier-Douady class of $\Gamma^{\prime} \rightarrow \Gamma \rightrightarrows M$. By construction we have a commutative diagram of groupoid morphisms

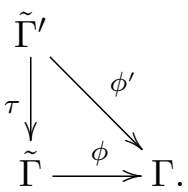

According to Proposition 4.15.(a), the Dixmier-Douady class $\boldsymbol{D} \boldsymbol{D}_{(\alpha)}$ is the cohomology class of the cocycle $\left[\Omega_{\alpha}\right]$ defined by the identity

$$
\operatorname{pr}(d \alpha+\partial \alpha)=\phi^{*}\left(\Omega_{\alpha}\right)
$$

Applying $\tau^{*}$ to Equation (20), we get

$$
\begin{aligned}
\tau^{*} \operatorname{pr}(d \alpha+\partial \alpha) & =\tau^{*} \phi^{*}\left(\Omega_{\alpha}\right) \\
\operatorname{pr}(d+\partial) \tau^{*} \alpha & =\phi^{\prime *}\left(\Omega_{\alpha}\right) \\
(d+\partial) \operatorname{pr} \tau^{*} \alpha & =\phi^{\prime *}\left(\Omega_{\alpha}\right) .
\end{aligned}
$$

Therefore, by Proposition 4.15, $\boldsymbol{D} \boldsymbol{D}_{\left(\alpha^{\prime}\right)}=\left[\Omega_{\alpha}\right]=\boldsymbol{D} \boldsymbol{D}_{(\alpha)}$.

Since $G$ is compact its center is the quotient $\left(Z_{0}(G) \times C\right) / N$, where $Z_{0}(G)$ is the connected component of $1_{G}$ in $Z(G)$ and $C, N$ are finite. We fix an isomorphism of Lie groups $Z_{0}(G) \cong S^{1} \times \cdots \times S^{1}$ (with $n$-factors). We thus obtain isomorphisms $Z(\mathfrak{g}) \cong \mathbb{R} e_{1} \oplus \cdots \oplus \mathbb{R} e_{n}$ and $H^{3}([Z(G) \rightarrow 1]) \cong$ $\mathbb{R} d t_{1} \oplus \cdots \oplus \mathbb{R} d t_{n}$. Let $\operatorname{pr}_{i}: Z(\mathfrak{g}) \rightarrow \mathbb{R} e_{i}(i=1, \ldots, n)$ be the natural projection.

Lemma 4.26. We have $\operatorname{pr}_{i}\left(\boldsymbol{C} \boldsymbol{C}_{\Phi}\right)=\boldsymbol{C} \boldsymbol{C}_{\Phi}\left(d t_{i}\right)$ in $H^{3}(\Gamma)$.

Proof. Let $\left(\xi_{1}, \ldots, \xi_{n}\right)$ be the dual basis of $\left(e_{1}, \ldots, e_{n}\right)$ in $Z(\mathfrak{g})^{*}$. According to [25], the generator $d t_{i}$ is the left invariant vector field $\xi_{i}^{L} \in \Omega^{1}(Z(G)) \subset$ $\Omega^{3}([Z(G) \rightarrow 1])$ associated to $\xi_{i}$. The Lemma follows.

Proposition 4.27. We have $\boldsymbol{C} \boldsymbol{C}_{\Phi}=\boldsymbol{D} \boldsymbol{D}_{\left(\alpha^{\prime}\right)}$

Proof. By linearity and Lemma 4.24, it is sufficient to prove that for all $i=1, \ldots, n$, one has

$$
\operatorname{pr}_{i}\left(\boldsymbol{D} \boldsymbol{D}_{(\theta)}\right)=\operatorname{pr}_{i}\left(\boldsymbol{C} \boldsymbol{C}_{\Phi}\right)=\boldsymbol{C} \boldsymbol{C}_{\Phi}\left(d t_{i}\right) \quad(\text { by Lemma 4.26). }
$$

The proof of Equation (21) is similar to that of Theorem 4.23. 
Proof of Theorem 4.18. By Proposition 4.25 and Proposition 4.27 we obtain

$$
C C_{\Phi}=D D_{\left(\alpha^{\prime}\right)}=D D_{(\alpha)}
$$

and Theorem 4.18 follows.

Example 4.28. Let $G$ be a simple compact Lie group and let $L G$ denote its loop group. Suppose that the Lie algebra $\mathfrak{g}$ of $G$ is endowed with $m$ invariant non-degenerate bilinear symmetric forms $\langle-,-\rangle_{i}(i=1, \ldots, m)$ and assume that the Lie algebra 2-cocycle $\beta \in \Lambda^{2}\left(L \mathfrak{g}^{*}\right) \otimes \mathbb{R}^{m}$ defined by

$$
\beta(X, Y)=\left(\frac{1}{2 \pi} \int_{0}^{2 \pi}\left\langle X(s), Y^{\prime}(s)\right\rangle_{1} d s, \ldots, \frac{1}{2 \pi} \int_{0}^{2 \pi}\left\langle X(s), Y^{\prime}(s)\right\rangle_{m} d s\right)
$$

is integral on every factor (i.e. the associated closed 2-form is). It thus gives rise to a central extension

$$
T^{m} \rightarrow \widetilde{L G} \rightarrow L G
$$

of the loop group by a torus $T^{m}$ of dimension $m$. The extension being central, the adjoint action of $\widetilde{L G}$ on its Lie algebra $\widetilde{L \mathfrak{g}}=L \mathfrak{g} \oplus \mathbb{R}^{m}$ descends to an action on $L \mathfrak{g}$ (which is compatible with the adjoint action of $L G$ on $L \mathfrak{g}$ ). Hence we have a central $T^{m}$-extension

$$
(\widetilde{L G} \times L \mathfrak{g} \rightrightarrows L \mathfrak{g}) \longrightarrow(L G \times L \mathfrak{g} \rightrightarrows L \mathfrak{g})
$$

of the associated transformation Lie groupoids. Since $(L G \times L \mathfrak{g} \rightrightarrows L \mathfrak{g})$ is Morita equivalent ${ }^{8}$ to the transformation groupoid $G \times G \rightrightarrows G$ (where $G$ acts on itself by conjugation), the extension (22) defines a central $T^{m}$-gerbe on $G \times G \rightrightarrows G$.

We compute the universal characteristic class of this central gerbe using the Dixmier-Douady class as follows. Note that the coadjoint action of $\widetilde{L G}$ on $\widetilde{L \mathfrak{g}}$ also restricts to $L \mathfrak{g}^{*}$ (identified with the affine hyperplane $\{(x, 1, \ldots$, 1) s.t. $x \in L \mathfrak{g}\})$. Let $\alpha=\alpha_{1} \oplus \cdots \oplus \alpha_{m} \in \Omega^{1}(\widetilde{L G} \times L \mathfrak{g}) \otimes \mathbb{R}^{m}$ be the direct sum of the dual (using on each coordinate of $\mathbb{R}^{m}$ the identification of $L \mathfrak{g}$ with $L \mathfrak{g}^{*}$ given by the form $\langle,\rangle_{i}$ ) of the restriction of the Liouville 1-form on $\widetilde{L G} \times \widetilde{L g}$ to $\Omega^{1}\left(\widetilde{L G} \times L \mathfrak{g}^{*}\right)$. By Proposition 4.15 and Lemma 4.26 , we obtain that the Dixmier-Douady class of the gerbe is a sum $\boldsymbol{D} \boldsymbol{D}_{(\alpha)}=\left[\Omega_{\alpha_{1}}\right] \oplus \cdots \oplus$

\footnotetext{
${ }^{8}$ The equivalence is induced by the projection $\left(\mathrm{ev}_{0}, \mathrm{Hol}\right)$, where $\mathrm{ev}_{0}$ is the evaluation map which takes a loop in $G$ to its value at 0 and $\mathrm{Hol}: L \mathfrak{g} \rightarrow G$ is the holonomy.
} 
$\left[\Omega_{\alpha_{m}}\right]$. By computation (see [9, Proposition 3.2]), we see that each class $\left[\Omega_{\alpha_{i}}\right]$ is equal in $H^{3}(G \times G \rightrightarrows G)$ to the class of $\left[b_{i}+\lambda_{i}\right]$ where $\lambda_{i}$ is the bi-invariant 3 -form coresponding to $\frac{1}{12}\langle-,[-,-]\rangle_{i} \in \Lambda^{3} \mathfrak{g}^{*}$ and

$$
\begin{aligned}
b_{i} & =-\frac{1}{2}\left[\left\langle\operatorname{Ad}_{x} g^{*}\left(\theta_{M C}\right), g^{*}\left(\theta_{M C}\right)\right\rangle_{i}+\left\langle g^{*}\left(\theta_{M C}\right), x^{*}\left(\theta_{M C}+\theta_{M C}^{r}\right)\right\rangle_{i}\right] \\
& \in \Omega^{2}(G \times G) \subset \Omega^{3}(G \times G \rightrightarrows G),
\end{aligned}
$$

where $\theta_{M C}$ and $\theta_{M C}^{r}$ are respectively the left and right Maurer-Cartan forms and $(g, x)$ denotes the coordinates on $G \times G$.

Since $G$ is a simple compact group, each $\langle-,-\rangle_{i}$ is an integer multiple

$$
\langle-,-\rangle_{i}=a_{i}\langle-,-\rangle_{\text {bas }}
$$

of the basic form of $G$. Hence $\left[b_{i}+\lambda_{i}\right]=a_{i}\left[b_{\text {bas }}+\lambda_{\text {bas }}\right]$ (where $b_{\text {bas }}, \lambda_{\text {bas }}$ are defined as above). Further, $\left[b_{\text {bas }}+\lambda_{\text {bas }}\right]$ is precisely an integral generator of $H^{3}(G \times G \rightrightarrows G)$.

It follows that the characteristic class of the gerbe is

$$
\boldsymbol{D} \boldsymbol{D}_{(\alpha)}=a_{1} \oplus \cdots \oplus a_{m} \in \mathbb{R}^{m}
$$

\section{Acknowledgments}

The authors are grateful to André Haefliger, Jim Stasheff, Urs Schreiber, Danny Stevenson, Ping Xu and the referee for many useful discussions and suggestions and are beholden to Damien Broka for contributing improvements to Section 3.2 .

This research was supported by the European Union through the FP6 Marie Curie R.T.N. ENIGMA (contract number MRTN-CT-2004-5652).

\section{References}

[1] Paolo Aschieri, Luigi Cantini and Branislav Jurčo, Nonabelian bundle gerbes, their differential geometry and gauge theory. Comm. Math. Phys., 254 (2005), no. 2, 367-400. MR2117631 (2005k:53022)

[2] John C. Baez and Aaron D. Lauda, Higher-dimensional algebra. V. 2-groups. Theory Appl. Categ., 12 (2004), 423-491. MR2068521 (2005m:18005)

[3] John C. Baez and Urs Schreiber, Higher gauge theory. Categories in algebra, geometry and mathematical physics, Contemp. Math., Vol. 
431, Amer. Math. Soc., Providence, RI, 2007, pp. 7-30. MR2342821 (2008k: 53099)

[4] John C. Baez and Danny Stevenson, The classifying space of a topological 2-group. Algebraic topology, Abel Symp., Vol. 4, Springer, Berlin, 2009, pp. 1-31. MR2597732 (2011h:55027)

[5] John C. Baez, Danny Stevenson, Alissa S. Crans and Urs Schreiber, From loop groups to 2-groups. Homology, Homotopy Appl., 9 (2007), no. 2, 101-135. MR2366945 (2009c:22022)

[6] Tobias Keith Bartels, Higher gauge theory: 2-bundles. Ph.D. thesis, University of California, Riverside, 2006.

[7] Kai Behrend, Grégory Ginot, Behrang Noohi and Ping Xu, String topology for stacks. Astérisque (2012), no. 343, xiv+169. MR2977576

[8] Kai Behrend and Ping Xu, Differentiable stacks and gerbes. J. Symplectic Geom., 9 (2011), no. 3, 285-341. MR2817778（2012k:53045)

[9] Kai Behrend, Ping Xu and Bin Zhang, Equivariant gerbes over compact simple Lie groups. C. R. Math. Acad. Sci. Paris, 336 (2003), no. 3, 251256. MR1968268 (2004i:58026)

[10] Kai A. Behrend, On the de Rham cohomology of differential and algebraic stacks. Adv. Math., 198 (2005), no. 2, 583-622. MR2183389 (2006g:14036)

[11] Jean Bénabou, Introduction to bicategories. Reports of the Midwest Category Seminar, Springer, Berlin, 1967, pp. 1-77. MR0220789 (36 \#3841)

[12] Lawrence Breen, Bitorseurs et cohomologie non abélienne. The Grothendieck Festschrift, Vol. I, Progr. Math., Vol. 86, Birkhäuser Boston, Boston, MA, 1990, pp. 401-476. MR1086889 (92m:18019)

[13] Lawrence Breen, On the classification of 2-gerbes and 2-stacks. Astérisque (1994), no. 225, 160. MR1301844 (95m:18006)

[14] Lawrence Breen, Tannakian categories. Motives (Seattle, WA, 1991), Proc. Sympos. Pure Math., Vol. 55, Amer. Math. Soc., Providence, RI, 1994, pp. 337-376. MR1265536 (95b:18009)

[15] Ronald Brown and Kirill C. H. Mackenzie, Determination of a double Lie groupoid by its core diagram. J. Pure Appl. Algebra, 80 (1992), no. 3, 237-272. MR1170713 (93g:55022) 
[16] M. Bullejos, E. Faro and V. Blanco, A full and faithful nerve for 2-categories. Appl. Categ. Structures, 13 (2005), no. 3, 223-233. MR2167791 (2006e:18009)

[17] Shiing-Shen Chern and Yi-Fone Sun, The imbedding theorem for fibre bundles. Trans. Amer. Math. Soc., 67 (1949), 286-303. MR0032996 $(11,378 c)$

[18] Paul Dedecker, Sur la cohomologie non abélienne. I. Canad. J. Math., 12 (1960), 231-251. MR0111021 (22 \#1888)

[19] Paul Dedecker, Sur la cohomologie non abélienne. II. Canad. J. Math., 15 (1963), 84-93. MR0143218 (26 \#778)

[20] Daniel Dugger and Daniel C. Isaksen, Topological hypercovers and $\mathbb{A}^{1}$-realizations. Math. Z., 246 (2004), no. 4, 667-689. MR2045835 (2005d:55026)

[21] Johan L. Dupont, Curvature and characteristic classes. Lecture Notes in Mathematics, Vol. 640, Springer-Verlag, Berlin-New York, 1978. MR0500997 (58 \#18477)

[22] John W. Duskin, Simplicial matrices and the nerves of weak $n$ categories. I. Nerves of bicategories. Theory Appl. Categ., 9 (2001/02), 198-308, CT2000 Conference (Como). MR1897816 (2003f :18005)

[23] Ezra Getzler, Lie theory for nilpotent $L_{\infty}$-algebras. Ann. of Math. (2), 170 (2009), no. 1, 271-301. MR2521116 (2010g:17026)

[24] G. Ginot and B. Noohi, Group actions on stacks and applications to equivariant string topology for stacks. ArXiv e-prints (2012).

[25] Grégory Ginot and Ping Xu, Cohomology of Lie 2-groups. Enseign. Math. (2), 55 (2009), no. 3-4, 373-396. MR2583784 (2011b:22004)

[26] Jean Giraud, Cohomologie non abélienne. Springer-Verlag, Berlin-New York, 1971, Die Grundlehren der mathematischen Wissenschaften, Band 179. MR0344253 (49 \#8992)

[27] André Haefliger, private communication.

[28] André Haefliger, Groupoids and foliations. Groupoids in analysis, geometry, and physics (Boulder, CO, 1999), Contemp. Math., Vol. 282, Amer. Math. Soc., Providence, RI, 2001, pp. 83-100. MR1855244 (2002m:57038) 
[29] André Henriques, Integrating $L_{\infty}$-algebras. Compos. Math., 144 (2008), no. 4, 1017-1045. MR2441255 (2011h:18021)

[30] Michel Hilsum and Georges Skandalis, Morphismes K-orientés d'espaces de feuilles et fonctorialité en théorie de Kasparov (d'après une conjecture d'A. Connes). Ann. Sci. École Norm. Sup. (4), 20 (1987), no. 3, 325-390. MR925720 (90a:58169)

[31] Nigel Hitchin, Lectures on special Lagrangian submanifolds. Winter School on Mirror Symmetry, Vector Bundles and Lagrangian Submanifolds (Cambridge, MA, 1999), AMS/IP Stud. Adv. Math., Vol. 23, Amer. Math. Soc., Providence, RI, 2001, pp. 151-182. MR1876068 (2003f : 53086)

[32] Masaki Kashiwara and Pierre Schapira, Categories and sheaves. Grundlehren der Mathematischen Wissenschaften [Fundamental Principles of Mathematical Sciences], Vol. 332, Springer-Verlag, Berlin, 2006. MR2182076 (2006k:18001)

[33] Camille Laurent-Gengoux, Mathieu Stiénon and Ping Xu, Non-abelian differentiable gerbes. Adv. Math., 220 (2009), no. 5, 1357-1427. MR2493616 (2010c:53039)

[34] Camille Laurent-Gengoux and Friedrich Wagemann, Obstruction classes of crossed modules of Lie algebroids and Lie groupoids linked to existence of principal bundles. Ann. Global Anal. Geom., 34 (2008), no. 1, 21-37. MR2415176 (2010b:17023)

[35] Tom Leinster, Higher operads, higher categories. London Mathematical Society Lecture Note Series, Vol. 298, Cambridge University Press, Cambridge, 2004. MR2094071 (2005h:18030)

[36] David Li-Bland and Pavol Ševera, Integration of exact Courant algebroids. Electron. Res. Announc. Math. Sci., 19 (2012), 58-76. MR2970717

[37] Zhang-Ju Liu, Alan Weinstein and Ping Xu, Manin triples for Lie bialgebroids. J. Differential Geom., 45 (1997), no. 3, 547-574. MR1472888 (98f : 58203)

[38] Marco Mackaay and Roger Picken, Holonomy and parallel transport for abelian gerbes. Adv. Math., 170 (2002), no. 2, 287-339. MR1932333 (2004a:53052) 
[39] Kirill C. H. Mackenzie, General theory of Lie groupoids and Lie algebroids. London Mathematical Society Lecture Note Series, vol. 213, Cambridge University Press, Cambridge, 2005. MR2157566 (2006k: 58035)

[40] Rajan Amit Mehta and Xiang Tang, From double Lie groupoids to local Lie 2-groupoids. Bull. Braz. Math. Soc. (N.S.), 42 (2011), no. 4, 651681. MR2861783

[41] Rajan Amit Mehta and Xiang Tang, Symplectic structures on the integration of exact Courant algebroids. ArXiv e-prints (2013).

[42] John W. Milnor and James D. Stasheff, Characteristic classes. Princeton University Press, Princeton, N. J.; University of Tokyo Press, Tokyo, 1974, Annals of Mathematics Studies, No. 76. MR0440554 (55 \#13428)

[43] I. Moerdijk, Lie groupoids, gerbes, and non-abelian cohomology. KTheory, 28 (2003), no. 3, 207-258. MR2017529 (2005b:58024)

[44] I. Moerdijk and J. Mrčun, Introduction to foliations and Lie groupoids. Cambridge Studies in Advanced Mathematics, Vol. 91, Cambridge University Press, Cambridge, 2003. MR2012261 (2005c:58039)

[45] M. K. Murray, Bundle gerbes. J. London Math. Soc. (2), 54 (1996), no. 2, 403-416. MR1405064 (98a:55016)

[46] Behrang Noohi, Notes on 2-groupoids, 2-groups and crossed modules. Homology, Homotopy Appl., 9 (2007), no. 1, 75-106. MR2280287 (2007m: 18006)

[47] Jean Pradines, Morphisms between spaces of leaves viewed as fractions. Cahiers Topologie Géom. Différentielle Catég., 30 (1989), no. 3, 229246. MR1029626 (91h:57018)

[48] Dorette A. Pronk, Groupoid representations for sheaves on orbifolds. Ph.D. thesis, Universiteit Utrecht, 1995.

[49] Dorette A. Pronk, Etendues and stacks as bicategories of fractions. Compositio Math., 102 (1996), no. 3, 243-303. MR1401424 (97d: 18011)

[50] Hisham Sati, Urs Schreiber and Jim Stasheff, $L_{\infty}$-algebra connections and applications to String- and Chern-Simons n-transport. Quantum field theory, Birkhäuser, Basel, 2009, pp. 303-424. MR2742762 (2012a:53043) 
[51] Graeme Segal, Classifying spaces and spectral sequences. Inst. Hautes Études Sci. Publ. Math., (1968), no. 34, 105-112. MR0232393 (38 \#718)

[52] Y. Sheng and C. Zhu, Higher Extensions of Lie Algebroids and Application to Courant Algebroids. ArXiv e-prints (2011).

[53] James D. Stasheff, H-spaces and classifying spaces: foundations and recent developments. Algebraic topology (Proc. Sympos. Pure Math., Vol. XXII, Univ. Wisconsin, Madison, Wis., 1970), Amer. Math. Soc., Providence, R.I., 1971, pp. 247-272. MR0321079 (47 \#9612)

[54] Norman Steenrod, The topology of fibre bundles. Princeton Landmarks in Mathematics, Princeton University Press, Princeton, NJ, 1999, Reprint of the 1957 edition, Princeton Paperbacks. MR1688579 (2000a:55001)

[55] Ross Street, The algebra of oriented simplexes, J. Pure Appl. Algebra 49 (1987), no. 3, 283-335. MR920944 (89a:18019)

[56] Charles A. Weibel, An introduction to homological algebra. Cambridge Studies in Advanced Mathematics, Vol. 38, Cambridge University Press, Cambridge, 1994. MR1269324 (95f:18001)

[57] Alan Weinstein and Ping Xu, Extensions of symplectic groupoids and quantization. J. Reine Angew. Math., 417 (1991), 159-189. MR1103911 (92k: 58094)

[58] Christoph Wockel, Principal 2-bundles and their gauge 2-groups. Forum Math., 23 (2011), no. 3, 565-610. MR2805195 (2012d:55023)

[59] Jesse Wolfson, Descent for n-bundles. Adv. Math., 288 (2016), 527-575. MR3436392

[60] Chenchang Zhu, n-groupoids and stacky groupoids. Int. Math. Res. Not. IMRN (2009), no. 21, 4087-4141. MR2549951 (2011c:18006) 
Institut de Mathématiques de Jussieu-Paris Rive Gauche UPMC - SORBONNE UNIVERSITÉS - PARIS 6 75252 PARIS CEDEX 05, FrANCE

E-mail address: gregory.ginot@imj-prg.fr

Department of Mathematics, Pennsylvania State University UNIVERSITY PARK, PA 16802, USA

E-mail address: stienon@psu.edu

Received May 28, 2013 
